Ana Claudia Rodrigues lopes Amaral de Souza

\title{
Modelo de predição para o uso de insulina em gestantes diagnosticadas com diabetes gestacional pela glicemia de jejum
}

Dissertação apresentada à Faculdade de Medicina da Universidade de São Paulo para obtenção do título de Mestre em Ciências

Programa de Obstetrícia e Ginecologia

Orientadora: Profa. Dra. Rossana Pulcineli Vieira Francisco

São Paulo 
Dados Internacionais de Catalogação na Publicação (CIP)

Preparada pela Biblioteca da

Faculdade de Medicina da Universidade de São Paulo

Creprodução autorizada pelo autor

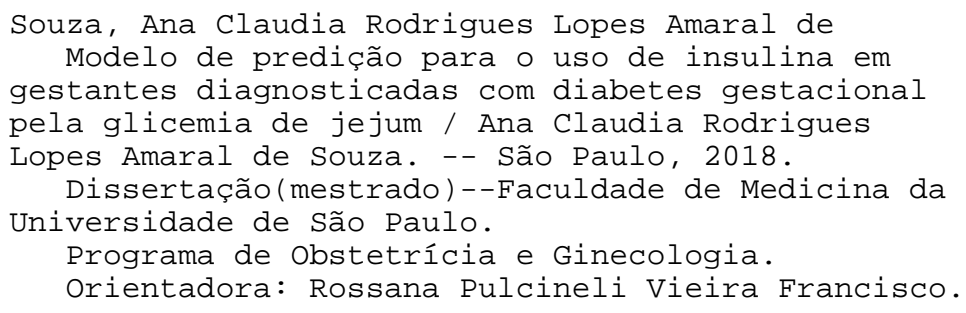

Descritores: 1.Diabetes gestacional 2.Glicemia de jejum 3.Insulina 4.Modelos logísticos

5. Nomogramas 6.Gravidez

$\mathrm{USP} / \mathrm{FM} / \mathrm{DBD}-286 / 18$ 
Dedico esta dissertação...

À minha avó Luzia. Meu norte, maior exemplo e porto seguro. 


\section{AGRADECIMENTOS}

Esta dissertação de mestrado nasceu da proposta de realização de uma monografia durante a residência médica e influenciou intensamente minhas escolhas profissionais até este momento, proporcionando-me aprendizados e novos sonhos, de uma maneira que eu não poderia imaginar ao dar os primeiros passos deste trabalho. Sua conclusão representa completar uma jornada desafiadora e a certeza de que tive os melhores companheiros de viagem, aos quais quero expressar minha profunda gratidão e carinho.

Agradeço a Deus por ser tão generoso, com todas as oportunidades e graças que me concede, por me guiar por todo o caminho, iluminar minhas escolhas e, especialmente, por me sustentar nas dificuldades.

À minha mãe, Leonor, pelo dom da vida e inspiração (in memoriam). Aos meus pais, Arthur e Oliete, por sempre me incentivarem a buscar o meu melhor e compreenderem a minha ausência em tantos fins de semana, para que eu pudesse me dedicar a este projeto. Ao meu irmão, Arthur, por dar-me apoio incondicional, por ter tornado a vida em São Paulo menos cinza com sua companhia e por auxiliar-me nas dificuldades digitais. À minha família minha avó, meus tios e meus primos - por torcerem por mim e serem sempre um refúgio e fortaleza. Amo muito vocês.

Aos meus queridos amigos - Ana Luiza Tonisi, Daniel Igami, Danielle Domingues, Débora Oriá, Laura Muzzi, Luiza Riccio, Marina Andres, Marina Gonzales, Renata Mendes e Rodolpho Truffa - presentes da residência para a vida toda. Obrigada por colocarem sorrisos em cada etapa desta trajetória, tornando-a infinitamente mais agradável, por me incentivarem, dividirem angústias e compartilharem tantas alegrias comigo.

Ao Prof. Dr. Marcelo Zugaib, pela oportunidade de ser recebida na Clínica Obstétrica do Hospital das Clínicas da Faculdade de Medicina da Universidade de São Paulo. Serviço de excelência, que me proporcionou e continua a me proporcionar tanto aprendizado. 
À Profa. Dra. Rossana Pulcineli Vieira Francisco, minha orientadora, pelo incentivo para que este trabalho fosse mais do que uma monografia, por me proporcionar descobrir a paixão pelo ensino e pela carreira acadêmica quando eu não vislumbrava essa possibilidade, pelo exemplo de dedicação e sensatez, por sempre estar presente e ensinar-me o poder de dar qualidade ao tempo.

À Dra. Rafaela Alkmin da Costa, que me guiou desde os primeiros passos deste trabalho até agora, que tornou a angústia de uma residente de primeiro ano uma pergunta científica, e tem sido um farol luminoso durante todos estes anos. Companheira constante nesta trajetória, foi um privilégio poder aprender tanto com quem admiro profundamente, pela profissional e ser humano que é, e por quem nutro imenso carinho e amizade. Muito obrigada!

Meu sincero agradecimento aos queridos colegas do setor de Endocrinopatias. À Dra. Cristiane Paganotti, por sua dedicação, disponibilidade e amizade diária. À Dra. Tatiana Assunção, que se fez presente mesmo distante e contribuiu brilhantemente para 0 desenvolvimento de nossa calculadora digital. Aos queridos Dra. Fernanda Cristina Mikami, Dr. Rodrigo Codarin e Dra. Veridiana Franco, por todas as oportunidades de compartilhar ideias e experiências.

A todos os docentes e assistentes da Clínica Obstétrica, que constroem a excelência desse centro diariamente, ensinaram-me tanto e continuam a ensinar, e são responsáveis pela formação que me orgulho imensamente de ter. Indescritível o prazer de poder conviver e compartilhar tanto com vocês. Meu carinho e gratidão a cada um.

À Agatha Rodrigues, por sua genialidade em conduzir a estatística deste trabalho, pelas conversas inspiradoras e pela amizade que construímos durante este percurso.

A todos os funcionários da clínica: equipe de enfermagem, administrativo e informática, por diariamente colaborarem para que a pesquisa em nosso serviço seja possível. Agradeço de maneira especial à 
enfermeira Ana Maria Sousa, por sua doçura, prestatividade diária e por tornar os dias mais harmoniosos, e à secretária Lucinda Pereira, por toda a sua paciência, pelo incentivo e por torcer por cada um na pós-graduação tão genuinamente.

Por fim agradeço carinhosamente às nossas gestantes, motivo principal deste estudo e do meu desejo de sempre buscar realizar uma assistência melhor. 
"No coração da ciência existe um equilíbrio essencial entre duas atitudes aparentemente contraditórias: uma abertura para ideias novas, por mais bizarras ou contrárias à intuição que sejam, e o exame cético mais implacável de todas as ideias, antigas e novas. É assim que verdades profundas são separadas de disparates profundos." 
Esta tese está de acordo com as seguintes normas, em vigor no momento desta publicação:

Referências: adaptado de International Committee of Medical Journals Editors (Vancouver).

Universidade de São Paulo. Faculdade de Medicina. Divisão de Biblioteca e Documentação. Guia de apresentação de dissertações, teses e monografias. Elaborado por Anneliese Carneiro da Cunha, Maria Julia de A. L. Freddi, Maria F. Crestana, Marinalva de Souza Aragão, Suely Campos Cardoso, Valéria Vilhena. $3^{a}$ ed. São Paulo: Divisão de Biblioteca e Documentação; 2011.

Abreviaturas dos títulos dos periódicos de acordo com List of Journals Indexed in Index Medicus. 


\section{SUMÁRIO}

LISTA DE ABREVIATURAS, SÍMBOLOS E SIGLAS

LISTA DE FIGURAS

LISTA DE GRÁFICOS

LISTA DE TABELAS

LISTA DE EQUAÇÕES

RESUMO

ABSTRACT

1. INTRODUÇÃO E REVISÃO DA LITERATURA ……........................... 1

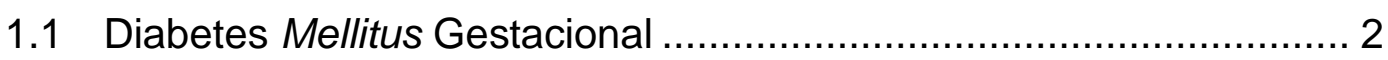

1.2 Breve histórico dos critérios para diagnóstico do diabetes

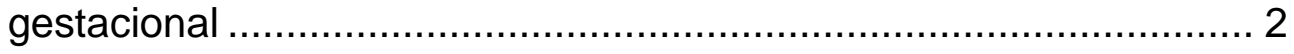

1.2.1 Estudo HAPO........................................................... 4

1.2.2 International Association of Diabetes and Pregnancy Study Groups ................................................................. 5

1.2.3 Consensos contemporâneos para o diagnóstico de DMG ...... 7

1.3 Impacto dos novos critérios diagnósticos ........................................ 8

1.4 Modelos de predição da gravidade do diabetes gestacional ............. 9

1.5 Justificativa do estudo .............................................................. 15

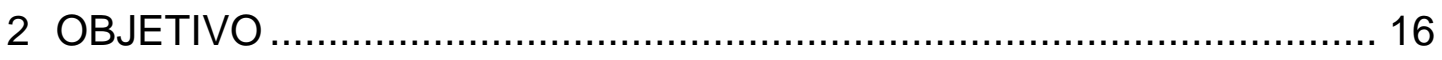

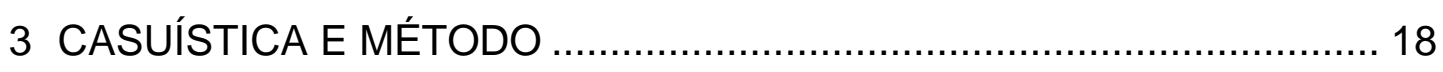

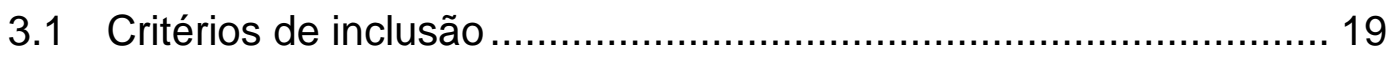

3.2 Rotina do atendimento pré-natal .............................................. 19

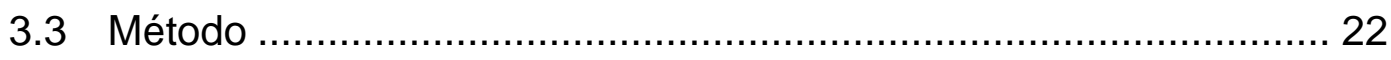

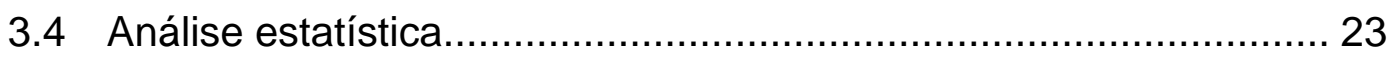

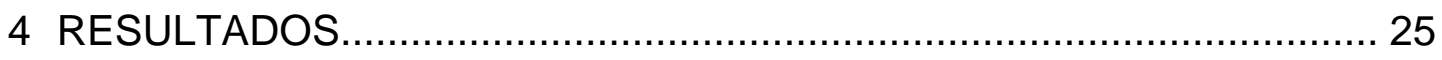

4.1 Caracterização da população e comparação dos grupos................. 26

4.2 Modelo de predição para o uso de insulina na gestação em mulheres diagnosticadas com diabetes gestacional pela

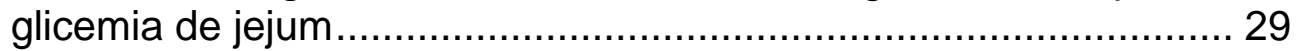

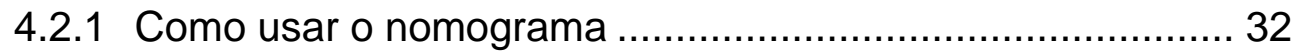

4.2.2 Desempenho clínico do modelo preditivo do uso de insulina. 
5 DISCUSSÃO

5.1 Características da população estudada ...................................... 40

5.2 Fatores de risco para uso de insulina em gestantes diagnosticadas com diabetes gestacional pela glicemia de jejum

5.3 Modelos de predição para o uso de insulina na gestação em mulheres diagnosticadas com diabetes gestacional ...................... 43

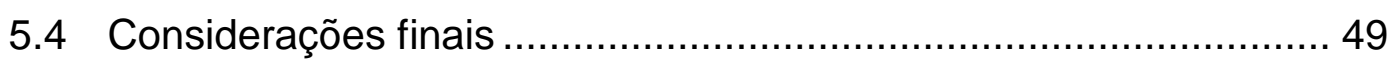

6 CONCLUSÃO

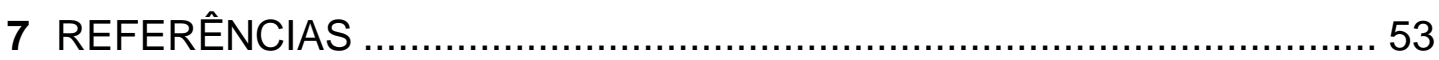




\section{LISTA DE ABREVIATURAS, SÍMBOLOS E SIGLAS}

$A C O G$

ADA

$\mathrm{AF}$

AIG

AP

ATLANTIC DIP

DM2

DMG

DP

et al

FIGO

FMUSP

g

GIG

GJ

$\mathrm{H}$

HAPO

HC-FMUSP

HCMED

IADPSG

IC $95 \%$

IG

IGF-1

ILA

IMC

$\mathrm{kg}$

$\mathrm{kg} / \mathrm{m}^{2}$

$\mathrm{mg} / \mathrm{dL}$

$\mathrm{N}$
The American College of Obstetricians and Gynecologists

American Diabetes Association

Antecedente familiar

Adequado para idade gestacional

Antecedente pessoal

The Atlantic Diabetes in Pregnancy

Diabetes mellitus tipo dois

Diabetes mellitus gestacional

Desvio padrão

et alia (e outros)

Federação Internacional de Ginecologia e Obstetrícia

Faculdade de Medicina da Universidade de São Paulo

Gramas

Grande para idade gestacional

Glicemia de jejum

Hora

Hyperglycemia and Adverse Pregnancy Outcome

Hospital das Clínicas da Faculdade de Medicina da Universidade de São Paulo

Sistema informatizado de laudos do laboratório central do Hospital das Clínicas

International Association of Diabetes and Pregnancy Study Groups

Intervalo de confiança de $95 \%$

Idade gestacional

Insulin Grouth Factor

Índice de líquido amniótico

Índice de massa corpórea

Quilos

Quilogramas por metro quadrado

Miligramas por decilitro

Número de indivíduos/ eventos 


$\begin{array}{ll}\text { NDDG } & \text { National Diabetes Data Group } \\ \text { OMS } & \text { Organização Mundial de Saúde } \\ \text { PIG } & \text { Pequeno para idade gestacional } \\ \text { RC } & \text { Razão de Chances } \\ \text { RN } & \text { Recém-nascido } \\ \text { ROC } & \text { Receiver Operating Characteristic Curve } \\ \text { SILOG } & \text { Sistema informatizado de laudos em obstetrícia e } \\ & \text { ginecologia } \\ \text { TOTG 100g } & \text { Teste oral de tolerância a glicose com } 100 \text { gramas } \\ \text { TOTG 75g } & \text { Teste oral de tolerância a glicose com } 75 \text { gramas } \\ \text { UTI } & \text { Unidade de terapia intensiva } \\ \text { VPN } & \text { Valor preditivo negativo } \\ \text { VPP } & \text { Valor preditivo positivo } \\ \% & \text { Por cento } \\ * & \text { Vezes } \\ + & \text { Mais } \\ \pm & \text { Mais ou menos } \\ > & \text { Maior que } \\ \geq & \text { Maior ou igual a } \\ < & \text { Menor que } \\ \leq & \text { Menor ou igual a }\end{array}$




\section{LISTA DE FIGURAS}

Figura 1 - Critérios diagnósticos do diabetes na gestação proposto pela International Association of Diabetes and Pregnancy Study Groups (IADPSG)

Figura 2 - Impresso disponibilizado pela Clínica Obstétrica do Hospital das Clínicas da Faculdade de Medicina da Universidade de São Paulo para as gestantes com diabetes gestacional para registro do controle glicêmico diário

Figura 3 - Fluxograma da seleção de pacientes para o estudo

Figura 4 - Nomograma para predição do uso de insulina em gestantes com diabetes gestacional diagnosticadas pela glicemia de jejum antes de 24 semanas de gestação.

Figura 5 - Exemplo do uso da calculadora digital disponível no website: obstetricia.hc.fm.usp.br/CalcGDM para predição do uso de insulina durante a gestação em mulheres diagnosticadas com diabetes gestacional pela glicemia de jejum 


\section{LISTA DE GRÁFICOS}

Gráfico 1 - Curva ROC do modelo de predição do uso de insulinoterapia em gestantes com diabetes gestacional diagnosticada pela glicemia de jejum - HC-FMUSP - São Paulo, 2012-2015. 


\section{LISTA DE TABELAS}

Tabela 1 - Evolução das propostas de critérios diagnósticos para diabetes gestacional de 1964 a 2008 (adaptado de Bhavadharin et al., 2016)

Tabela 2 - Protocolos mais comuns para o diagnóstico do diabetes gestacional (tabela adaptada do consenso da OMS de 2013)

Tabela 3 - Prevalência de DMG de acordo com os critérios diagnósticos adotados e a população estudada 8

Tabela 4 - Modelo de predição em que a soma dos pontos das variáveis maior ou igual a 4 prediz uso de farmacoterapia em diabetes gestacional leve (tabela adaptada de Mendez-Figueroa et al., 2014)

Tabela 5 - Modelo logístico de predição de insulinoterapia em gestantes com DMG proposto por Barnes et al., 2016, Austrália

Tabela 6 - Síntese da revisão de literatura de estudos que propõe modelos para predição de gravidade do diabetes gestacional

Tabela 7 - Características clínicas das gestantes com diabetes gestacional diagnosticadas pela glicemia de jejum, de acordo com o tratamento recebido - HC-FMUSP - São Paulo, 2012-2015 
Tabela 8 - Resultado do modelo de regressão logística multivariada para predição de insulinoterapia em gestantes com diabetes gestacional diagnosticada pela glicemia de jejum - HC-FMUSP - São Paulo, 2012-2015

Tabela 9 - Pontuação correspondente a cada variável do nomograma. 33

Tabela 10 - Desempenho do rastreamento para probabilidade do uso de insulina em gestantes com diabetes gestacional definido pelo modelo de regressão logística quando comparado com o uso real de insulina nas pacientes estudadas - HC-FMUSP - São Paulo, 2012-2015 


\section{LISTA DE EQUAÇÕES}

Equação 1- Probabilidade do uso de insulina na gestação em mulheres diagnosticadas com diabetes gestacional pela glicemia de jejum: ............................................................... 30 


\section{RESUMO}

Souza ACRLA. Modelo de predição para o uso de insulina em gestantes diagnosticadas com diabetes gestacional pela glicemia de jejum [dissertação]. São Paulo: Faculdade de Medicina, Universidade de São Paulo; 2018.

Objetivo: Avaliar os fatores de risco e propor um modelo para a predição da necessidade de insulina durante o tratamento de Diabetes Mellitus Gestacional (DMG) diagnosticado precocemente. Métodos: coorte retrospectiva de gestantes que foram diagnosticadas com DMG pela glicemia de jejum (GJ) anormal na primeira visita pré-natal e que receberam atendimento pré-natal em um hospital terciário de ensino em São Paulo, Brasil, entre 2012 e 2015. De acordo com a necessidade de insulinoterapia para atingir os alvos glicêmicos, as gestantes foram divididas em dois grupos (grupo Insulina ou grupo Dieta) e comparadas quanto a variáveis clínicas e laboratoriais. $O$ desempenho dessas variáveis na predição da necessidade de insulina para o tratamento do DMG foi identificado por modelo de regressão logística, e um nomograma foi criado para facilitar a interpretação clínica. Resultados: No total, foram incluídas 408 mulheres para análise. Entre elas, 135 (33\%) necessitaram de terapia com insulina. No modelo de regressão logística, idade materna, índice de massa corporal prégestacional, valor da GJ, história de DMG anterior e história familiar de diabetes foram variáveis independentes significativas para a predição da necessidade de insulina. Conclusão: O modelo de predição elaborado permitiu a construção de um nomograma e uma calculadora digital de fácil uso clínico para avaliar a necessidade de insulinoterapia em mulheres com diagnóstico precoce de DMG.

Descritores: Diabetes gestacional; Glicemia de jejum; Insulina; Modelos logísticos; Nomogramas; Gravidez. 


\begin{abstract}
Souza ACRLA. Prediction model for insulin need in women diagnosed with gestational diabetes by fasting blood glucose [dissertation]. São Paulo: "Faculdade de Medicina, Universidade de São Paulo"; 2018.

Objective: To evaluate risk factors and to propose a model for the prediction of insulin requirement during the treatment of early-diagnosed gestational diabetes mellitus (GDM). Methods: Retrospective cohort with chart review of pregnant women receiving antenatal care at a tertiary teaching hospital in São Paulo, Brazil, who were diagnosed with GDM by abnormal fasting blood glucose (FBG) at the first prenatal visit between 2012 and 2015. Groups were divided according to the requirement for insulin therapy to achieve blood glucose targets (insulin or diet group), the subjects were compared regarding clinical and laboratory variables. The performance of these variables in predicting insulin need for GDM treatment was identified by a logistic regression model, and a nomogram was created to facilitate clinical interpretation. Results: In total, 408 women were included for analysis. Among them, 135 (33\%) needed insulin therapy. In the logistic regression model, maternal age, pre-pregnancy body mass index, FBG value, prior GDM and family history of diabetes were significant independent variables for the prediction of insulin need. Conclusion: The prediction model found allowed the construction of a nomogram and digital calculator that is easy to use in order to evaluate the need for insulin therapy in women with early diagnosis of GDM.
\end{abstract}

Descriptors: Diabetes; Gestational; Fasting blood glucose; Insulin; Logistic models; Nomograms; Pregnancy. 


\section{INTRODUÇÃO E REVISÃO DA LITERATURA}

\subsection{Diabetes Mellitus Gestacional}

Diabetes mellitus gestacional (DMG) é definido pela presença de intolerância à glicose diagnosticada pela primeira vez durante a gestação ${ }^{(1)}$. Está associado às morbidades maternas e neonatais em curto e longo prazos $^{(2,3)}$, o que determina a importância de seu diagnóstico e tratamento. Sua prevalência tem aumentado na população mundial, fazendo com que seu manejo torne-se grande desafio para a saúde pública e incitando trabalhos como este, que se propõe a encontrar ferramentas que auxiliem nesse processo ${ }^{(4)}$.

\subsection{Breve histórico dos critérios para diagnóstico do diabetes gestacional}

O'Sullivan e Mahan foram os primeiros a propor, em 1964, um critério para o diagnóstico do DMG. O objetivo era identificar hiperglicemias relacionadas ao maior risco de mulheres persistirem com diabetes mellitus tipo II (DM2) após a gestação. Eram necessários dois ou mais valores glicêmicos alterados para o diagnóstico do DMG, e não foram analisados resultados perinatais adversos para a sua definição ${ }^{(5)}$.

No fim dos anos 1970, a possibilidade de ensaio enzimático para a análise da glicemia sérica motivou novos estudos para adaptação dos valores diagnósticos de referência. Desde então, novos critérios para o diagnóstico do DMG foram propostos, todos baseados no resultado de testes de sobrecarga glicêmica e que pretendiam identificar quais mulheres com hiperglicemia na gestação teriam maior risco de ser diagnosticada com 
DM2 após o puerpério. As variações entre os protocolos deviam-se ao número de etapas necessárias até o teste confirmatório, quantidade de glicose oferecida à gestante e número de valores alterados necessários para o diagnóstico.

Algumas dessas propostas diagnósticas estão demonstradas na Tabela 1, adaptada de Bhavadharin et al. (2016) ${ }^{(6)}$, a qual apresenta, além do critério diagnóstico inicial de 1964, a proposta de Carpenter \& Coustan em $1982{ }^{(7)}$, a recomendação da Organização Mundial da Saúde em $1999{ }^{(1)}$ e, por fim, o critério da Associação Americana de Diabetes de $2004^{\left({ }^{(8)}\right.}$. A diversidade de propostas demonstra que o diagnóstico do DMG vem sendo estudado, discutido e repensado há várias décadas, como demonstra a literatura.

Tabela 1 - Evolução das propostas de critérios diagnósticos para diabetes gestacional de 1964 a 2008 (adaptado de Bhavadharin et al., 2016) ${ }^{(6)}$

\begin{tabular}{|c|c|c|c|c|c|c|c|}
\hline \multirow{2}{*}{ Critério } & \multirow{2}{*}{ Ano } & \multirow{2}{*}{ Etapas } & \multirow{2}{*}{$\begin{array}{l}\text { Teste de } \\
\text { tolerância } \\
\text { à glicose } \\
\text { (g) }\end{array}$} & \multicolumn{4}{|c|}{$\begin{array}{l}\text { Glicemia sérica } \\
(\mathrm{mg} / \mathrm{dL})\end{array}$} \\
\hline & & & & jejum & $1 \mathrm{~h}$ & $2 \mathrm{~h}$ & $3 \mathrm{~h}$ \\
\hline O’Sullivan \& Mahan* (5) & 1964 & 2 & 100 & 90 & 165 & 145 & 125 \\
\hline Carpenter \& Coustan* ${ }^{\star(7)}$ & 1982 & 2 & 100 & 95 & 180 & 155 & 140 \\
\hline $\begin{array}{l}\text { Organização Mundial } \\
\text { da Saúde }(\text { OMS })^{\star \star(1)}\end{array}$ & 1999 & 1 & 75 & 126 & ----- & 140 & ----- \\
\hline $\begin{array}{l}\text { Associação Americana } \\
\text { de Diabetes (ADA)* }\end{array}$ & 2004 & 2 & 100 & 95 & 180 & 155 & 140 \\
\hline
\end{tabular}

g: gramas, h: hora

* necessários dois valores ou mais para o diagnóstico

** um valor alterado é suficiente para o diagnóstico 


\subsubsection{Estudo HAPO}

Como já exposto, desde 1964, o diagnóstico do DMG fundamentavase no risco do DM2 após o parto. Em 2008, uma mudança de referência para o diagnóstico foi apresentada. Diferente das propostas anteriores, um grande estudo multicêntrico chamado Hyperglycemia and Adverse Pregnancy Outcome (HAPO) analisou a relação entre hiperglicemia materna e desfechos perinatais desfavoráveis ${ }^{(2)}$.

Tratou-se de grande estudo multicêntrico que envolveu 25.505 gestantes selecionadas entre julho de 2000 e abril de 2006 em 15 centros hospitalares de nove países (norte-americanos, europeus, asiáticos e da Oceania). Tais gestantes foram submetidas ao teste oral de tolerância à glicose de $75 \mathrm{~g}$ (TOTG $75 \mathrm{~g}$ ) entre a $24^{\mathrm{a}}$ e a $32^{\mathrm{a}}$ semanas de gestação, analisados seus níveis glicêmicos em jejum, 1 e 2 horas após sobrecarga de glicose, e seus médicos não tiveram acesso a esses valores durante o prénatal.

Foram excluídas do estudo, por questões de segurança, aquelas com glicemia de jejum maior que $105 \mathrm{mg} / \mathrm{dL}$ e/ou glicemia duas horas após sobrecarga de glicose maior ou igual a $200 \mathrm{mg} / \mathrm{dL}$.

Considerou-se desfechos desfavoráveis primários: peso ao nascimento maior que o percentil 90 para idade gestacional, parto cesáreo, hipoglicemia neonatal e nível de peptídeo C no cordão umbilical maior que percentil 90 (hiperinsulinemia fetal).

Como desfechos secundários foram estudadas: prematuridade (parto antes de 37 semanas de gestação), distocia de ombro ou tocotraumatismos, necessidade de cuidado intensivo neonatal, hiperglicemia e pré-eclâmpsia.

$\mathrm{Na}$ análise dos resultados, encontrou-se que tanto as glicemias de jejum quanto as de uma ou duas horas após a sobrecarga são preditoras independentes para desfechos neonatais adversos e, dessa forma, um único valor anormal no teste foi identificado como suficiente para predizer 0 aumento do risco de complicações perinatais. As associações foram 
semelhantes entre os centros de estudos envolvidos, sugerindo aplicabilidade global.

Apesar das relações consistentes encontradas entre a hiperglicemia materna e os desfechos gestacionais desfavoráveis, os investigadores do estudo HAPO não fizeram recomendações específicas quanto aos critérios diagnósticos a serem adotados.

\subsubsection{International Association of Diabetes and Pregnancy Study Groups}

Diante dos resultados consistentes apresentados pelo estudo HAPO, a International Association of Diabetes and Pregnancy Study Groups (IADPSG), com representantes de múltiplas organizações de obstetrícia e diabetes, revisou as recomendações para o diagnóstico do diabetes gestacional e sugeriu a adoção de novos valores para o diagnóstico do DMG, pela primeira vez relacionados aos desfechos perinatais, e não ao risco materno para DM2 ${ }^{(9)}$.

O protocolo proposto recomendava o rastreio universal das gestantes, ou seja, que todas as mulheres sem diagnóstico prévio de diabetes, e não apenas aquelas com fatores de risco, deveriam ser submetidas ao TOTG 75 $g$ entre a $24^{\text {a }}$ e a $28^{a}$ semana de gestação. Para definição dos valores diagnósticos, foi considerada a Razão de Chances $(R C)$ de 1,75 dos valores apresentados pelo estudo HAPO. Dessa maneira, para o diagnóstico de DMG, deveria-se considerar:

- Glicemia de jejum maior ou igual a ( $\geq$ ) $92 \mathrm{mg} / \mathrm{dL}$

- 1 hora após sobrecarga $\geq 180 \mathrm{mg} / \mathrm{dL}$

- 2 horas após sobrecarga $\geq 153 \mathrm{mg} / \mathrm{dL}$

Reforçando-se que bastaria um valor alterado para o diagnóstico de DMG, sem necessidade de testes de triagem prévios positivos. 
A glicemia de jejum na primeira consulta pré-natal também passou a ser recomendada a todas as gestantes como uma forma de diagnosticar 0 diabetes gestacional, não dependendo de testes de tolerância à glicose confirmatórios posteriormente à sua realização.

Caso a glicemia de jejum fosse maior ou igual a $92 \mathrm{mg} / \mathrm{dL}$ e menor que $126 \mathrm{mg} / \mathrm{dL}$, dever-se-ia considerar o diagnóstico de diabetes, não sendo

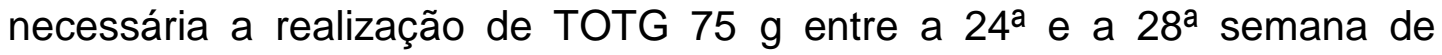
gestação. Caso o valor encontrado fosse maior ou igual a $126 \mathrm{mg} / \mathrm{dL}$, deveria ser considerado diagnóstico de diabetes pré-gestacional, à semelhança dos critérios usados para as mulheres não gestantes. Nesses casos, os cuidados durante o pré-natal são aos moldes das pacientes com diagnóstico de DM2.

O teste de sobrecarga entre a $24^{a}$ e a $28^{\underline{a}}$ semana estaria reservado, então, para aquelas gestantes cuja glicemia de jejum na primeira consulta de pré-natal fosse menor que $92 \mathrm{mg} / \mathrm{dL}$. O critério diagnóstico proposto pela IADPSG está exposto no fluxograma da Figura 1.

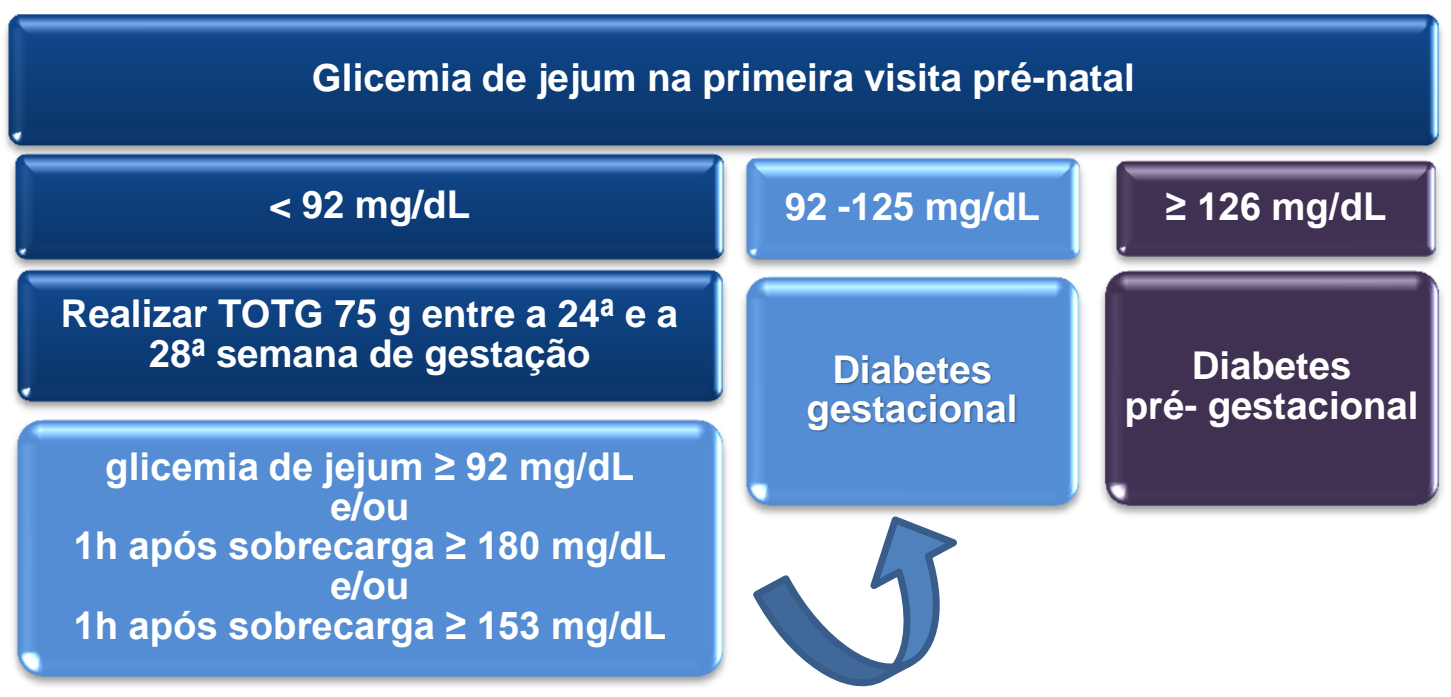

TOTG: teste oral de tolerância à glicose, g: gramas, h: hora, mg/dL: miligramas por decilitro

Figura 1 - Critérios diagnósticos do diabetes na gestação propostos pela International Association of Diabetes and Pregnancy Study Groups (IADPSG) ${ }^{(9)}$ 


\subsubsection{Consensos contemporâneos para o diagnóstico de DMG}

$\mathrm{Na}$ Tabela 2, adaptada do consenso da Organização Mundial da Saúde (OMS) de 2013 sobre diabetes gestacional ${ }^{(1)}$, está a comparação entre o novo critério diagnóstico proposto pela IAPSG ${ }^{(9)}$ e os demais critérios diagnósticos vigentes na época em que ele foi proposto. Entretanto, vale ressaltar que a própria OMS, em 2013, passou a recomendar os critérios propostos pela IADPSG. Ainda observa-se na Tabela 20 posicionamento das diferentes organizações após a publicação da IADPSG.

Tabela 2 - Protocolos mais comuns para o diagnóstico do diabetes gestacional (tabela adaptada do consenso da OMS de 2013) ${ }^{(1)}$

\begin{tabular}{|c|c|c|c|c|c|}
\hline Organização & $\begin{array}{l}\text { Glicemia } \\
\text { de jejum } \\
\text { (mg/dL) }\end{array}$ & $\begin{array}{c}\text { Teste de } \\
\text { sobrecarga } \\
\text { glicêmica }\end{array}$ & $\begin{array}{l}\text { Glicemia } \\
\text { após } \\
1 \text { hora } \\
\text { (mg/dL) }\end{array}$ & $\begin{array}{l}\text { Glicemia } \\
\text { após } \\
2 \text { horas } \\
\text { (mg/dL) }\end{array}$ & $\begin{array}{l}\text { Glicemia } \\
\text { após } \\
3 \text { horas } \\
\text { (mg/dL) }\end{array}$ \\
\hline OMS 1999*(1) & $\geq 126$ & $75 \mathrm{~g}$ & --- & $\geq 140$ & --- \\
\hline $\mathbf{A C O G} * *(10)$ & $\geq 95$ & $100 \mathrm{~g}$ & $\geq 180$ & $\geq 155$ & $\geq 140$ \\
\hline $\begin{array}{l}\text { Ministério da Saúde } \\
\text { - Brasil 2012 }\end{array}$ & $\geq 95$ & $75 \mathrm{~g}$ & $\geq 180$ & $\geq 155$ & ---- \\
\hline IADPSG* $^{*}$ & $\geq 92$ & $75 \mathrm{~g}$ & $\geq 180$ & $\geq 153$ & ---- \\
\hline OMS 2013*(1) & $\geq 92$ & $75 \mathrm{~g}$ & $\geq 180$ & $\geq 153$ & $\overline{----}$ \\
\hline FIGO* ${ }^{(4)}$ & $\geq 92$ & $75 \mathrm{~g}$ & $\geq 180$ & $\geq 153$ & ---- \\
\hline $\begin{array}{l}\text { Ministério da Saúde } \\
\text {-Brasil 2017* }\end{array}$ & $\geq 92$ & $75 \mathrm{~g}$ & $\geq 180$ & $\geq 153$ & ---- \\
\hline \multicolumn{6}{|c|}{$\begin{array}{l}\text { OMS= Organização Mundial da Saúde, ACOG= The American College of Obstetricians and } \\
\text { Gynecologists, IADPSG= International Association of Diabetes and Pregnancy Study Groups, FIGO= } \\
\text { Federação Internacional de Ginecologia e Obstetrícia, } \mathrm{mg} / \mathrm{dL}=\text { miligramas por decilitro } \\
\text { * um valor alterado é suficiente para o diagnóstico } \\
\text { ** necessários dois valores alterados ou mais para o diagnóstico } \\
\text { *** realizar teste de sobrecarga glicêmica se glicemia entre } 85 \mathrm{mg} / \mathrm{dL} \text { e } 125 \mathrm{mg} / \mathrm{dL} \text { ou presença de } \\
\text { fator de risco clínico. Necessários dois valores alterados ou mais para o diagnóstico. Se apenas um } \\
\text { valor alterado, repetir o teste com } 34 \text { semanas de gestação }\end{array}$} \\
\hline
\end{tabular}




\subsection{Impacto dos novos critérios diagnósticos}

Após a adoção dos critérios diagnósticos propostos pela IADPSG, foi constatado aumento significativo da prevalência do DMG ${ }^{(3)}$.

Em seu documento de 2013 sobre diabetes gestacional, a OMS estimou que $10 \%$ das gestantes, se avaliadas pelo critério diagnóstico assumido pela própria organização em 1999, apresentariam diabetes gestacional e que esse número aumentaria $50 \%$, ou seja, para $15 \%$ das gestações, se assumidos os novos critérios propostos pela IADPSG ${ }^{(1)}$.

Em 2001, estudo brasileiro com 4.977 gestantes ${ }^{(11)}$ demonstrou uma incidência de 7,2\% de DMG entre as brasileiras, utilizando-se o critério diagnóstico da OMS de 1999, e de 2,4\%, usando-se o critério da Associação Americana de Diabetes (ADA), ambos apresentados previamente na Tabela 1. Essa mesma população foi avaliada à luz do novo critério proposto pela IADPSG, e a incidência da doença entre essas gestantes aumentaria para $18 \%{ }^{(12)}$.

Estudo chinês demonstrou aumento de $200 \%$ no diagnóstico do DMG em população seguida, de 2008 a 2011, pelos critérios da ADA quando reclassificada pelos novos critérios da IADPSG ${ }^{(13)}$.

O aumento da prevalência do DMG demonstrada nos estudos citados está sintetizado na Tabela 3.

Tabela 3 - Prevalência de DMG de acordo com os critérios diagnósticos adotados e a população estudada

\begin{tabular}{llrrr}
\hline \multirow{2}{*}{ Estudo } & População & \multicolumn{3}{c}{ Prevalência DMG } \\
\cline { 2 - 5 } & & OMS 1999 & ADA & IADPSG \\
\hline OMS, 2013 ${ }^{(1)}$ & $\begin{array}{l}44.829 \text { - revisão de } \\
\text { literatura com 8 estudos }\end{array}$ & $10,0 \%$ & --- & $15 \%$ \\
Liao, 2014 ${ }^{(13)}$ & 5.360 chinesas & $7,2 \%$ & $11,7 \%$ & $24,5 \%$ \\
Trujillo, 2015 ${ }^{(12)}$ & 4.977 brasileiras & --- & $2,4 \%$ & $18 \%$ \\
\hline OMS = Organização & Mundial da Saúde, ADA = American Diabetes & Association, & IADPSG = \\
International Association of Diabetes and Pregnancy Study Groups & & &
\end{tabular}


Como demonstrado, é notável o aumento da incidência do DMG na população mundial quando se adotam os critérios diagnósticos propostos pela IADPSG. Esse aumento de incidência, associado ao impacto nos recursos necessários para seu tratamento adequado, tem levado obstetras e endocrinologistas a debater sobre os benefícios dos novos critérios diagnósticos e a buscar alternativas para a sua implantação.

\subsection{Modelos de predição da gravidade do diabetes gestacional}

Determinar quais gestantes apresentarão manifestações mais graves da doença tem sido motivo de estudos. Uma estratégia tem sido considerar que gestantes com DMG que exigem insulinoterapia para adequado controle glicêmico representam formas mais graves da doença. Sendo assim, determinar os fatores de risco para uso de insulina tem sido uma forma de identificar tais casos ${ }^{(14-16)}$.

Encontrar meios de estratificar esse risco permitiria direcionar de maneira mais adequada as orientações, os cuidados e o planejamento durante o pré-natal, priorizando casos mais graves nos locais em que os recursos são restritos, a fim de se evitar as repercussões da doença. Ao passo que aquelas com menor gravidade poderiam ser tranquilizadas por apresentarem formas mais leves da doença. A literatura é escassa em apresentar modelos de estratificação de risco para a insulinoterapia na gestação. A seguir serão apresentados os únicos quatro estudos que propõem modelos de predição da gravidade do DMG.

Sapienza et al., em 2010, apresentaram resultados de um estudo brasileiro com 294 gestantes diagnosticadas com DMG quando apresentavam dois ou mais valores alterados no TOTG $100 \mathrm{~g}$ realizado entre a 24a e a 34ª semana de gestação; adotando os critérios de diagnóstico da ADA (Tabela 1), propuseram modelo de predição do uso de insulina nessas gestantes. Demonstraram que obesidade pré-gestacional $(p=0,003)$, história familiar de diabetes $(p=0,008)$, número de valores alterados no TOTG $100 \mathrm{~g}$ 
$(p=0,003)$ e dosagem de hemoglobina glicada $(p<0,001)$ eram preditores independentes para formas mais graves de hiperglicemia na gestação, que demandaram uso de insulinoterapia para o controle metabólico ${ }^{(17)}$.

Considerando-se um ponto de corte de 0,3, seu modelo demonstrou acurácia de 75,2\% quando excluída a hemoglobina glicada da análise, o que seria compatível com a avaliação na primeira consulta pré-natal após o diagnóstico de DMG, e de $81,2 \%$ ao incluir a hemoglobina glicada no modelo, reavaliação possível na segunda consulta pré-natal com resultado do exame solicitado no momento do diagnóstico ${ }^{(17)}$.

Em 2011, Mendez-Figueroa et al. estudaram um subgrupo de gestantes com diabetes que chamaram de DMG leve (Mild GDM). Tratavase de gestantes diagnosticadas com diabetes gestacional pelo TOTG $100 \mathrm{~g}$ pelos critérios da ADA, mas que apresentavam a glicemia de jejum no exame menor que $95 \mathrm{mg} / \mathrm{dL}$, ou seja, apenas 2 ou 3 dos valores após a sobrecarga glicêmica alterados ${ }^{(18)}$.

Entre as 1.457 gestantes atendidas em seu serviço entre 2006 e 2011, 367 gestantes foram incluídas no estudo e 143 (38,9\%) delas necessitaram de insulina ou glibenclamida durante a gestação para adequado controle glicêmico. Em seu modelo multivariado de regressão logística, três variáveis mostraram-se preditoras independentes para farmacoterapia no DMG: níveis maiores da GJ no TOTG, menor idade gestacional do diagnóstico e maior IMC entre a 26 a a $30^{\text {a }}$ semana de gestação. Baseadas na razão de chances, cada uma das variáveis recebeu uma pontuação, como demonstrado na Tabela 4, que foi adaptada do trabalho em questão. Quando a soma dos pontos das variáveis fosse maior ou igual a 4, o modelo tinha a sua melhor relação entre sensibilidade e especificidade para predição do uso de insulina, com uma área abaixo da curva ROC de $0,7^{(18)}$. 
Tabela 4 - Modelo de predição em que a soma dos pontos das variáveis maior ou igual a 4 prediz uso de farmacoterapia em diabetes gestacional leve (tabela adaptada de Mendez-Figueroa et al., 2014) ${ }^{(18)}$

\begin{tabular}{lrrrr}
\hline & $\mathbf{0}$ & $\mathbf{1}$ & $\mathbf{2}$ & \multicolumn{1}{c}{$\mathbf{3}$} \\
\hline Glicemia de jejum (mg/dL) & $<80$ & ---- & $\geq 80$ & ---- \\
IMC, IG entre 26 e 30 semanas $\left(\mathbf{k g} / \mathbf{m}^{2}\right)$ & $\leq 28,4$ & $28,5-32,9$ & $\geq 33$ & ---- \\
IG do diagnóstico (semanas) & $>30$ & ---- & $25-30$ & $<25$ \\
\hline
\end{tabular}

IMC: índice de massa corpórea, IG: idade gestacional, $\mathrm{mg} / \mathrm{dL}$ : miligramas por decilitro, $\mathrm{kg} / \mathrm{m}^{2}$ : quilogramas por metro quadrado

Um modelo de predição de insulinoterapia apenas com parâmetros clínicos foi proposto em 2014 por Pertot et al. Entre 1995 e 2010, 3.009 gestantes com diabetes gestacional diagnosticado pelos critérios da Sociedade Australiana de Diabetes foram atendidas em hospital australiano (19). Entre elas, 1.535 (51\%) fizeram uso de insulinoterapia durante a gestação. A análise univariada demonstrou associação entre o uso de insulina durante a gestação e o diagnóstico do DMG antes de 25 semanas de gestação $(p<0,001)$, IMC pré-gestacional $(p<0,001)$, história familiar de diabetes $(p<0,001)$, valor da glicemia de jejum $(p<0,001)$, valor da glicemia uma hora após sobrecarga de glicose $(p<0,001)$, valor da hemoglobina glicada $(p<0,001)$ e idade materna $(p=0,02)$.

No modelo de regressão logística proposto, permaneceram como variáveis preditoras independentes para insulinoterapia 0 valor da hemoglobina glicada (OR 2,2), a história familiar de diabetes (OR 1,4), o valor da glicemia de jejum (OR 1,3), o valor da glicemia 1 hora após sobrecarga de insulina (OR 1,06) e o IMC (OR 1,04). Os autores propuseram que valores do modelo abaixo de $50 \%$ de risco para insulinoterapia representariam um grupo chamado "baixo risco", entre $60 \%$ e $80 \%$, "médio risco" e, acima de $80 \%$, "alto risco". Com os valores de corte propostos, o modelo apresentaria $66 \%$ de sensibilidade e $37 \%$ de especificidade. Das gestantes que fizeram uso de insulina, 59,2\% foram classificadas como 
baixo risco por esse modelo, sendo que 33,3\% delas com probabilidade estimada de até $10 \%{ }^{(19)}$.

Em 2016, novo modelo de predição de insulinoterapia foi proposto por outro estudo australiano, dessa vez com 3.317 mulheres atendidas entre 1992 e 2015 e diagnosticadas com DMG pelos critérios da Sociedade Australiana de Diabetes de 1991 e revistos em $1998^{(20)}$. Nesse estudo, as gestantes foram separadas em dois grupos: o grupo que controlou a hiperglicemia apenas com dieta e outro que associou insulina à dieta para controle da hiperglicemia. As variáveis com significância estatística na análise univariada foram dicotomizadas e testadas em modelo de regressão logística. Sete delas se mostraram preditoras independentes para uso de insulina na gestação: idade materna maior que 30 anos, história familiar de diabetes, IMC pré-gestacional $\geq 30 \mathrm{~kg} / \mathrm{m}^{2}$, história prévia de DMG, diagnóstico precoce de DMG (antes de 24 semanas de gestação), GJ $\geq 95$ $\mathrm{mg} / \mathrm{dL}$ e hemoglobina glicada no diagnóstico $\geq 5,5 \%{ }^{(21)}$.

Tal modelo considerou que o número de variáveis presentes era capaz de predizer risco de insulinoterapia, de forma que definiram três grupos: com nenhuma ou apenas uma variável presente, com duas a cinco variáveis presentes e com seis ou sete variáveis presentes. Não foi avaliado se cada variável influenciava de forma diferente o resultado final do modelo, o qual apresentou área abaixo da curva ROC de 0,7. Esse modelo está demonstrado na Tabela 5 adaptada do estudo original ${ }^{(21)}$. 
Tabela 5 - Modelo logístico de predição de insulinoterapia em gestantes com DMG proposto por Barnes et al., 2016, Austrália ${ }^{(21)}$

Fatores preditores independentes de insulinoterapia (as variáveis quantitativas foram dicotomizadas): idade $>30$ anos, obesidade pré-gestacional $\geq 30 \mathrm{~kg} / \mathrm{m}^{2}$, idade gestacional < 24 semanas, glicemia de jejum no diagnóstico > 95 $\mathrm{mg} / \mathrm{dL}$, hemoglobina glicada no diagnóstico > 5,5\%, AF de DM2 e AP de DMG

Uso do modelo: As variáveis são avaliadas segundo estarem presentes ou não e o número de variáveis presentes interpretado da seguinte forma:

\begin{tabular}{lll}
\hline Baixo risco & Risco intermediário & Alto risco \\
$0-1$ variável & $2-5$ variáveis & $6-7$ variáveis
\end{tabular}

DMG: diabetes mellitus gestacional, $\mathrm{kg} / \mathrm{m}^{2}$ : quilogramas por metro quadrado, $\mathrm{AF}$ : antecedente familiar, DM2: diabetes mellitus tipo 2, AP: antecedente pessoal

Os quatro estudos que propõem modelos de predição de risco para farmacoterapia no diabetes gestacional estão sintetizados na Tabela 6 . 
Tabela 6 - Síntese da revisão de literatura de estudos que propõe modelos para predição de gravidade do diabetes gestacional

\begin{tabular}{|c|c|c|c|c|c|}
\hline Estudo & $\begin{array}{c}\text { Critério } \\
\text { diagnóstico }\end{array}$ & $\mathbf{n}$ & $\begin{array}{c}\% \\
\text { farmacoterapia }\end{array}$ & $\begin{array}{l}\text { Preditores independentes de } \\
\text { farmacoterapia }\end{array}$ & Desempenho do modelo \\
\hline $\begin{array}{l}\text { Sapienza et al., } 2010 \\
\text { Brasil }^{(17)}\end{array}$ & ADA & 294 & $117(40 \%)$ & $\begin{array}{l}\text { Obesidade pré-gestacional, história familiar de } \\
\text { diabetes, número de valores no TOTG } 100 \mathrm{~g} \\
\text { alterados, valor da HbA1C }\end{array}$ & $\begin{array}{l}\text { Para corte } 0,3 \text {, acurácia } \\
66,4 \% \text { (sem HbA1c) } \\
65,6 \% \text { (com HbA1c) }\end{array}$ \\
\hline $\begin{array}{l}\text { Mendez-Figueroa et al., } \\
2014 \text {, Estados Unidos }\end{array}$ & ADA & 367 & $143(39 \%)$ & $\begin{array}{l}\text { Idade gestacional no diagnóstico, valor da GJ } \\
\text { no TOTG } 100 \mathrm{~g} \text {, IMC entre } 26 \text { e } 30 \text { semanas } \\
\text { de gestação }\end{array}$ & $\begin{array}{l}\text { Área sob a curva ROC } 0,7 \\
\text { Para corte } 4,75,8 \% \\
\text { sensibilidade e } 51,5 \% \\
\text { especificidade }\end{array}$ \\
\hline $\begin{array}{l}\text { Pertot et al., 2014, } \\
\text { Australia }^{(19)}\end{array}$ & ADIPS 1998 & 3.009 & $1.535(51 \%)$ & $\begin{array}{l}\text { História familiar de diabetes, IMC, valor de GJ, } \\
\text { valor da } 1^{\mathrm{a}} \text { hora da sobrecarga, valor } \mathrm{HbA} 1 \mathrm{C}\end{array}$ & $\begin{array}{l}\text { Para corte } 0,5,66 \% \\
\text { sensibilidade e } 37 \% \\
\text { especificidade }\end{array}$ \\
\hline $\begin{array}{l}\text { Barnes et al.., 2016, } \\
\text { Australia }{ }^{(21)}\end{array}$ & ADIPS 1998 & 3.317 & $1.302(39 \%)$ & $\begin{array}{l}\text { Idade materna }>30 \text { anos, IMC pré-gestacional } \\
\geq 30 \mathrm{~kg} / \mathrm{m}^{2}, I G<24 \text { semanas, valor da GJ } \geq \\
95 \mathrm{mg} / \mathrm{dL} \text {, valor da HbA1C no diagnóstico } \geq \\
5,5 \% \text {, história familiar de diabetes, História } \\
\text { prévia de DMG }\end{array}$ & Área sob a curva ROC 0,7 \\
\hline
\end{tabular}

TOTG: teste oral tolerância a glicose, HbA1C: hemobglobina glicada, GJ: glicemia de jejum, IMC: índice de massa corpórea, IG: idade gestacional, mg/dL: miligramas por decilitro, $\mathrm{kg} / \mathrm{m}^{2}$ : quilogramas por metro quadrado, DMG: diabetes mellitus gestacional, ADA: American Diabetes Association, ADIPS: Australasian Diabetes in Pregnancy Society

\begin{tabular}{|c|c|c|c|c|c|c|}
\hline \multirow{2}{*}{ Critério } & \multirow{2}{*}{ Etapas } & \multirow{2}{*}{ TOTG (g) } & \multicolumn{4}{|c|}{ Valor glicemia sérica } \\
\hline & & & Jejum & $1 \mathrm{~h}$ & $2 \mathrm{~h}$ & $3 \mathrm{~h}$ \\
\hline ADA $2004^{(8)}$ & 2 & 100 & 95 & 180 & 155 & 140 \\
\hline ADIPS $1998^{(25)}$ & 1 & 75 & 100 & 100 & 144 & -- \\
\hline
\end{tabular}


Até o presente momento nenhum dos modelos propostos para estratificar a gravidade do diabetes na gestação estudou especificamente as gestantes diagnosticadas com DMG precocemente pela glicemia de jejum alterada na primeira visita pré-natal, um grupo relativamente novo de gestantes e que passou a ser identificado apenas após adoção dos critérios diagnósticos propostos pela IADPSG.

Embora os estudos existentes indiquem que o diagnóstico precoce de DMG antes de 24 semanas de gestação é um fator de risco para a necessidade de insulina durante o tratamento, muito pouco se sabe sobre esse grupo específico de mulheres.

\subsection{Justificativa do estudo}

A Federação Internacional de Ginecologia e Obstetrícia (FIGO) aprovou recentemente os critérios da IADPSG para o diagnóstico do DMG ${ }^{(4)}$. No entanto, diante do impasse do aumento da prevalência do DMG e da escassez de recursos financeiros, encoraja fortemente cada país a encontrar estratégias para adequar o diagnóstico e o tratamento ao orçamento local para a saúde.

O diagnóstico do DMG no início da gestação implica em grande aumento de sua prevalência e, consequentemente, maior uso de recursos do sistema de saúde para tratá-lo adequadamente. Porém, poucos estudos na literatura debruçaram-se sobre esse grupo específico de gestantes, aquelas com diagnóstico de DMG pela GJ no início da gestação.

Assim sendo, indo ao encontro da recomendação da FIGO e buscando preencher essa lacuna da literatura, o presente estudo dedica-se a identificar os fatores de risco e propor um modelo para prever a gravidade do DMG, tendo como parâmetro a necessidade de insulina durante seu tratamento, nesse grupo de gestantes ainda pouco estudado, diagnosticado precocemente pela glicemia de jejum na primeira consulta de pré-natal. 


\section{OBJETIVO}

O objetivo deste estudo é avaliar os fatores preditores do uso de insulina em gestantes diagnosticadas com diabetes gestacional pela glicemia de jejum antes de 24 semanas de gestação e verificar a possibilidade de predizer a necessidade de insulinoterapia nessas pacientes utilizando um modelo de predição. 


\section{CASUÍSTICA E MÉTODO}

O presente estudo foi realizado no Setor de Endocrinopatias na Gestação da Divisão de Clínica Obstétrica do Hospital das Clínicas da Faculdade de Medicina da Universidade de São Paulo (HC-FMUSP). Tratase de estudo retrospectivo, do tipo coorte, que envolveu gestantes acompanhadas no serviço, cuja primeira consulta pré-natal ocorreu no período de 01/01/2012 a 31/12/2015.

\subsection{Critérios de inclusão}

Foram elegíveis gestações únicas com até 24 semanas de idade gestacional, de mulheres diagnosticadas com diabetes gestacional por alteração da glicemia de jejum (glicemia $\geq 92 \mathrm{mg} / \mathrm{dL}$ e $<126 \mathrm{mg} / \mathrm{dL}$ ), de acordo com o consenso da IADPSG. Foram considerados, ainda, como critérios de elegibilidade para o estudo:

- Ausência de perda de seguimento pré-natal no serviço;

- Registros completos no prontuário para avaliação do desfecho primário;

- Gestação que evoluiu além de 20 semanas; e

- Não utilização de corticoterapia para maturação pulmonar fetal.

\subsection{Rotina do atendimento pré-natal}

Em 2011, o setor de Endocrinopatias na Gestação do HC-FMUSP adotou os critérios diagnósticos para DMG propostos pela IADPSG e, desde então, todas as gestantes diagnosticadas por esses critérios recebem tratamento ${ }^{(22) .}$ 
As mulheres diagnosticadas com DMG são encaminhadas primeiramente para um grupo de orientação com equipe multidisciplinar composta por um médico, uma enfermeira e uma nutricionista, para receber informações sobre o que é DMG, as possíveis repercussões na gestação e como são feitos o acompanhamento e o tratamento.

Nessa oportunidade, as gestantes são orientadas a respeito da realização de atividade física, respeitando-se as contraindicações obstétricas aos exercícios. São incentivadas a realizar caminhadas por cerca de trinta minutos três a cinco vezes por semana.

Quanto à dieta, são aconselhadas a ingerir de 1.800 a 2.200 calorias por dia, divididas em seis refeições (café da manhã, lanche matinal, almoço, lanche da tarde, jantar e ceia), consistindo de aproximadamente $50 \%$ de carboidratos, $30 \%$ a $35 \%$ de gordura e $15 \%$ a $20 \%$ de proteína. As gestantes são instruídas a preferir alimentos de baixo índice glicêmico, como carboidratos integrais.

São orientadas ainda a realizar o automonitoramento de medidas de glicemia capilar quatro vezes por dia, a saber: no jejum e uma hora após o café da manhã, almoço e jantar. As medidas de glicemia capilar são aferidas com um glicosímetro que é disponibilizado à gestante na primeira consulta após o diagnóstico e registradas em impresso demonstrado na Figura 2. Tais medidas são conferidas e registradas em sistema informatizado pelo obstetra em todas as visitas pré-natais. 


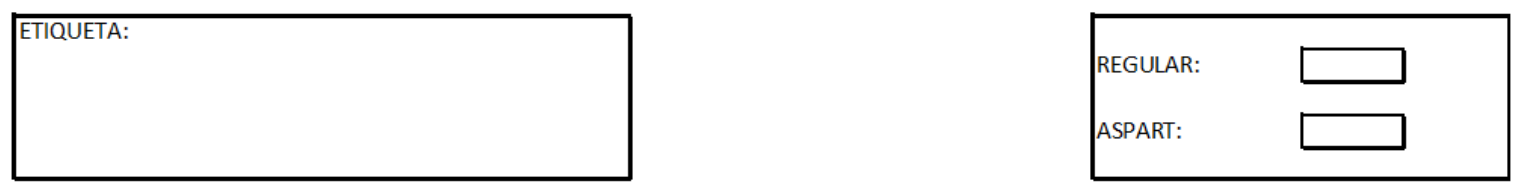

\begin{tabular}{|c|c|c|c|c|c|c|c|c|c|c|c|c|c|}
\hline \multicolumn{8}{|c|}{ DEXTROS } & \multicolumn{6}{|c|}{ INSULINA } \\
\hline DATA & $\begin{array}{l}\text { JEJUM } \\
\text { (<95) }\end{array}$ & $\begin{array}{c}01 \text { HORA } \\
\text { APÓS CAFÉ } \\
(<140) \\
\end{array}$ & $\begin{array}{l}\text { ANTES DO } \\
\text { ALMOÇO } \\
(<100)\end{array}$ & $\begin{array}{l}1 \text { 1 H APÓS } \\
\text { ALMOÇO } \\
(<40) \\
\end{array}$ & $\begin{array}{c}\text { ANTES DO } \\
\text { JANTAR } \\
(<100) \\
\end{array}$ & $\begin{array}{c}1 \text { 1 HAPÓS } \\
\text { JANTAR } \\
(<140)\end{array}$ & $\begin{array}{c}03 \mathrm{H} \\
\text { MADRUGADA } \\
(<100)\end{array}$ & $\begin{array}{l}\text { NPH } \\
\text { CEDO }\end{array}$ & $\begin{array}{c}\text { REG. } \\
\text { PRÉ CAFÉ }\end{array}$ & $\begin{array}{l}\text { REG. PRÉ } \\
\text { ALMOÇO }\end{array}$ & $\begin{array}{c}\text { NPH } \\
\text { ALMOÇO } \\
\end{array}$ & $\begin{array}{l}\text { REG. PRÉ } \\
\text { JANTAR }\end{array}$ & $\begin{array}{l}\text { NPH } \\
22 \mathrm{HS} \\
\end{array}$ \\
\hline & & & & & & & & & & & & & \\
\hline & & & & & & & & & & & & & \\
\hline & & & & & & & & & & & & & \\
\hline & & & & & & & & & & & & & \\
\hline & & & & & & & & & & & & & \\
\hline & & & & & & & & & & & & & \\
\hline & & & & & & & & & & & & & \\
\hline & & & & & & & & & & & & & \\
\hline & & & & & & & & & & & & & \\
\hline & & & & & & & & & & & & & \\
\hline & & & & & & & & & & & & & \\
\hline & & & & & & & & & & & & & \\
\hline & & & & & & & & & & & & & \\
\hline
\end{tabular}

FONTE: Clínica Obstétrica do Hospital das Clínicas da Faculdade de Medicina da Universidade de São Paulo

Figura 2 - Impresso disponibilizado pela Clínica Obstétrica do Hospital das Clínicas da Faculdade de Medicina da Universidade de São Paulo para as gestantes com diabetes gestacional para registro do controle glicêmico diário

O intervalo entre as visitas de seguimento pré-natal é programado de acordo com a idade gestacional, o tipo de tratamento e o controle glicêmico, podendo variar de 1 a 4 semanas. Aquelas em uso de insulina, com níveis mais altos de glicemia ou idade gestacional mais avançada são avaliadas com maior frequência.

Quando os alvos glicêmicos (jejum < 95 mg/dL e 1 hora pós-refeição < $140 \mathrm{mg} / \mathrm{dL}$ ) não são alcançados com a dieta em pelo menos 70\% das medidas aferidas, a terapia com insulina é associada ao tratamento dietético. Quando há sinais de repercussão fetal e o peso fetal estimado está acima do percentil 70, a insulinoterapia é indicada se menos que $80 \%$ das medidas estiverem dentro do alvo terapêutico. A importância da dieta adequada e do 
controle de ganho de peso é constantemente reforçada nas visitas prénatais.

\subsection{Método}

Os dados foram obtidos por meio de acesso ao sistema informatizado do setor de Endocrinopatias na Gestação da Clínica Obstétrica do HCFMUSP, acesso aos prontuários das gestantes, acesso ao Sistema Informatizado de Internação da Clínica Obstétrica do HC-FMUSP, acesso ao Sistema Informatizado de Laudos em Obstetrícia e Ginecologia (SILOG) e ao sistema informatizado de laudos do Laboratório Central do HC-FMUSP (HCMED).

As gestantes estudadas foram divididas em dois grupos, de acordo com o desfecho primário de interesse, que foi o uso ou não de insulina para controle glicêmico:

a) Dieta: grupo de gestantes cuja hiperglicemia foi controlada com dieta

b) Insulina: grupo de gestantes que requisitou insulinoterapia associada à dieta para controle da hiperglicemia

Esses grupos foram comparados quanto aos fatores sociodemográficos, clínicos e laboratoriais, a saber:

- Idade (anos);

- $\quad$ Primigesta (sim, não);

- IMC pré-gestacional ou IMC do primeiro trimestre, para aquelas que não souberam referir o peso prévio à gestação $\left(\mathrm{kg} / \mathrm{m}^{2}\right)$;

- Categoria do IMC pré-gestacional (baixo peso, normal, sobrepeso, obesidade);

- Ganho de peso total na gestação $(\mathrm{kg})$; 
- Antecedente familiar de diabetes (sim, não);

- Antecedente pessoal de diabetes gestacional prévio (sim, não);

- Antecedente de macrossomia fetal (filho anterior com peso ao nascer $\geq 4.000 \mathrm{~g}$ ) (sim, não);

- Tabagismo (sim, não);

- Antecedente de hipertensão arterial crônica (sim, não);

- Idade gestacional do diagnóstico (semanas);

- Valor da glicemia de jejum que determinou o diagnóstico (mg/dL); e

- Idade gestacional do início da insulinoterapia no grupo em que foi necessário associar a insulina ao tratamento (semanas).

Foram realizados testes de regressão logística para avaliar quais fatores foram associados à necessidade do uso de insulina.

\subsection{Análise estatística}

Foram realizadas análises univariadas comparando o grupo Dieta com o grupo Insulina. As variáveis quantitativas foram comparadas utilizando o teste $t$ de Student. As associações entre variáveis qualitativas foram avaliadas pelo teste Qui-quadrado ou teste exato de Fisher, quando apropriado.

Para as variáveis que se mostraram estatisticamente diferentes entre os grupos, um modelo de regressão logística multivariado foi construído, e seu desempenho foi avaliado para predizer a probabilidade do uso de insulina para o tratamento da hiperglicemia.

O método automático Stepwise foi considerado para a seleção de variáveis, resultando em um modelo final com aquelas que foram selecionadas. Para quantificar a influência de cada preditor sobre a chance de uso de insulina, a razão de chances (odds ratio - OR) e seu respectivo intervalo de confiança de 95\% (IC 95\%) foram estimados. 
Com base nesse modelo, foi construído um nomograma para representação do efeito de cada variável na predição da probabilidade de insulinoterapia.

Para avaliar o desempenho do nosso modelo, realizamos a análise da curva ROC (Receiver Operating Characteristic Curve) e avaliamos o valor preditivo positivo (VPP), o valor preditivo negativo (VPN) e a acurácia para diferentes cortes de especificidade para o modelo.

Para validar a capacidade do modelo preditivo, considerou-se o método de validação cruzada leave one out para estimar a sua precisão ${ }^{(23)}$. Essa é uma alternativa para validação do modelo utilizando-se a mesma população estudada. Nesse processo, é retirada uma observação, ajusta-se o modelo e classifica-se a observação com base no modelo sem ela. $O$ processo é repetido para todos os casos e avalia-se a acurácia do modelo proposto.

Os dados foram analisados usando o pacote estatístico SPSS (IBM SPSS Statistics para Windows, versão 2.0) e o pacote "Caret" do Software R versão 3.0.2 (R Core Team 2016) ${ }^{(24)}$. Os testes foram considerados significativos quando $p<0,05$. 


\section{RESULTADOS}

\subsection{Caracterização da população e comparação dos grupos}

Foram identificadas 438 mulheres com gestação única diagnosticadas pela GJ antes de 24 semanas de gestação e atendidas na Clínica Obstétrica do HC-FMUSP entre janeiro de 2012 e dezembro de 2015.

Não foram incluídas na análise quatro gestantes cuja gravidez não evoluiu além de 20 semanas, três que usaram corticoide durante a gravidez para maturação pulmonar, seis mulheres que perderam o seguimento prénatal e 17 que tinham registros médicos incompletos.

Dessa forma, 408 gestantes foram incluídas no estudo, compondo a amostra final, como exposto na Figura 3. 


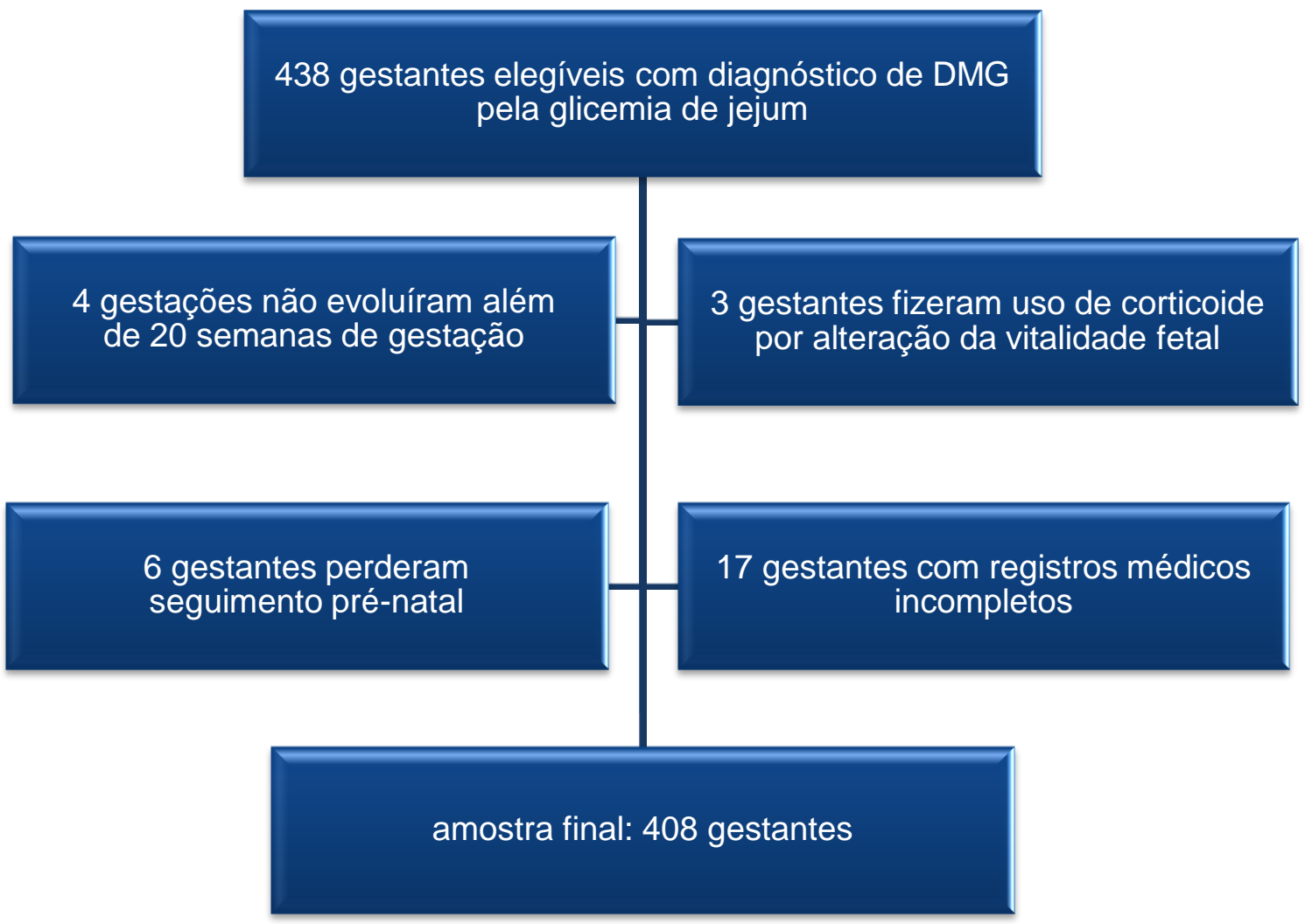

DMG: diabetes mellitus gestacional

Figura 3 - Fluxograma da seleção de pacientes para o estudo

Assim, a população desse estudo caracterizou-se por 408 gestantes com idade média de $33 \pm 6,0$ anos, que iniciaram o pré-natal com 10,6 \pm 4,2 semanas de gestação em média e composta de 98 (24\%) gestantes primigestas. Das 408 gestantes, 197 (48,3\%) eram obesas e 117 (28,7\%) estavam com sobrepeso. Por ser um serviço de pré-natal de alto risco, a população estudada caracterizou-se por 113 (27,7\%) hipertensas crônicas, $17(4,2 \%)$ asmáticas, $10(2,5 \%)$ com síndrome de ovários policísticos e 6 $(1,5 \%)$ lúpicas.

Das 408 gestantes selecionadas, 135 (33,1\%) necessitaram de terapia com insulina, e o medicamento foi prescrito em média com 26,0 $\pm 7,1$ semanas de gestação. Observamos que 52/135 (38,5\%) das pacientes que precisaram de insulinoterapia iniciaram o uso de insulina antes de 24 semanas de gestação. 
Em comparação com o grupo Dieta, aquelas que necessitaram de insulina eram mais velhas $(p=0,001)$, não eram prímiparas, frequência $(p=0,038)$, apresentavam maior IMC pré-gestacional $(p=0,001)$, mais frequentemente tinham história familiar de diabetes $(p=0,005)$, história de DMG em gestação anterior ( $p<0,001)$, macrossomia fetal prévia $(p=0,007)$ e diagnóstico de hipertensão crônica $(p=0,006)$. Tinham ainda níveis mais elevados de GJ no diagnóstico $(p<0,001)$. Os resultados da análise univariada estão descritos na Tabela 7.

Tabela 6 - Características clínicas das gestantes com diabetes gestacional diagnosticadas pela glicemia de jejum, de acordo com 0 tratamento recebido - HC-FMUSP - São Paulo, 2012-2015

\begin{tabular}{|c|c|c|c|}
\hline & $\begin{array}{c}\text { Dieta } \\
(\mathrm{n}=273)\end{array}$ & $\begin{array}{c}\text { Insulina } \\
(n=135)\end{array}$ & Valor $p$ \\
\hline Idade materna (anos), média $\pm \mathrm{DP}$ & $32,0 \pm 6,1$ & $34,1 \pm 5,7$ & $0,001^{1}$ \\
\hline Primigesta, $\mathrm{n}(\%)$ & $74(27,1)$ & $24(17,8)$ & $0,038^{2}$ \\
\hline IMC pré-gestacional $\left(\mathrm{kg} / \mathrm{m}^{2}\right)$, média \pm DP & $29,8 \pm 6,9$ & $32,2 \pm 6,4$ & $<0,001^{1}$ \\
\hline Categoria do IMC pré-gestacional & & & $0,001^{2}$ \\
\hline Obesidade $\left(\mathrm{IMC}>29,9 \mathrm{~kg} / \mathrm{m}^{2}\right)^{3}, \mathrm{n}(\%)$ & $114(41,8)$ & $80(59,3)$ & \\
\hline Sobrepeso (IMC>24,9 kg/m²), n (\%) & $88(32,2)$ & $39(28,9)$ & \\
\hline Normal/baixo peso (IMC <25 kg/ m²), n (\%) & $71(26,0)$ & $16(11,9)$ & \\
\hline $\begin{array}{l}\text { Ganho de peso durante a gestação } \\
(\mathrm{kg}) \text {, média } \pm \text { DP }\end{array}$ & $8,0 \pm 7,3$ & $8,3 \pm 6,6$ & $0,648^{1}$ \\
\hline Antecedente familiar de diabetes, $n(\%)$ & $159(58,5)$ & $98(72,6)$ & $0,005^{2}$ \\
\hline Antecedente de DMG ${ }^{5}, \mathrm{n}(\%)$ & $17(6,2)$ & $32(23,7)$ & $<0,001^{2}$ \\
\hline Antecedente de macrossomia fetal, $\mathrm{n}(\%)$ & $16(7,2)$ & $19(16,8)$ & $0,007^{2}$ \\
\hline Tabagismo, n (\%) & $19(7,0)$ & $15(11,2)$ & $0,153^{2}$ \\
\hline Hipertensão arterial crônica, n (\%) & $64(23,4)$ & $49(36,3)$ & $0,006^{2}$ \\
\hline $\begin{array}{l}\text { Idade gestacional no diagnóstico do DMG } \\
\text { (semanas), média } \pm \text { DP }\end{array}$ & $10,5 \pm 4,1$ & $10,9 \pm 4,4$ & $0,430^{1}$ \\
\hline $\begin{array}{l}\text { Glicemia de jejum no diagnóstico } \\
\text { (mg/dL), média } \pm \mathrm{DP}\end{array}$ & $96,8 \pm 5,2$ & $101,1 \pm 7,8$ & $<0,001^{1}$ \\
\hline $\begin{array}{l}\text { Idade gestacional de início da } \\
\text { insulinoterapia (semanas), média } \pm \text { DP }\end{array}$ & - & $26,0 \pm 7,1$ & \\
\hline $\begin{array}{l}\text { Insulinoterapia antes de } 24 \text { semanas de } \\
\text { gestação, n (\%) }\end{array}$ & - & $52(38,5)$ & - \\
\hline
\end{tabular}

DP: desvio padrão, IMC: índice de massa corpórea, $\mathrm{kg} / \mathrm{m}^{2}$ : quilogramas por metro quadrado, $\mathrm{kg}$ : quilogramas, DMG: diabetes mellitus gestacional, $\mathrm{mg} / \mathrm{dL}$ : miligramas por decilitro, ${ }^{1}$. Teste $\mathrm{t}$ de Student; ${ }^{2}$. Teste Qui quadrado; $p<0.05$. 


\subsection{Modelo de predição para o uso de insulina na gestação em} mulheres diagnosticadas com diabetes gestacional pela glicemia de jejum

As variáveis clínicas e laboratoriais com diferença estatisticamente significativa entre os grupos, identificadas na análise univariada, foram selecionadas para ajuste no modelo de regressão logística. As variáveis selecionadas foram:

- $\quad$ Idade materna (anos);

- $\quad$ Primigesta (sim, não);

- $\quad$ IMC pré-gestacional $\left(\mathrm{kg} / \mathrm{m}^{2}\right)$;

- Categoria do IMC pré-gestacional (baixo peso, normal, sobrepeso, obesidade);

- História familiar de diabetes (sim, não);

- DMG em gestação prévia (sim, não);

- História de recém-nascido macrossômico prévio (sim, não);

- Hipertensão arterial crônica (sim, não); e

- Glicemia de jejum no diagnóstico (mg/dL).

No modelo de regressão logística, idade materna, IMC prégestacional, história familiar de diabetes, DMG anterior e nível de glicemia de jejum no diagnóstico mostraram-se preditores independentes, e a fórmula a seguir foi encontrada para predição de insulinoterapia em gestantes diagnosticadas com diabetes gestacional pela glicemia de jejum. 
Equação 1 - Probabilidade do uso de insulina na gestação em mulheres diagnosticadas com diabetes gestacional pela glicemia de jejum

$$
\begin{gathered}
\operatorname{Exp}[-12,230+(0,818, \text { se obesidade })+(0,456, \text { se sobrepeso })+(0,582, \text { se AF de DM })+ \\
(1,032, \text { se AP de DMG })+(0,088 * \text { valor } G J)+(0,054 * \text { idade })] \\
\frac{1+\exp [-12,230+(0,818, \text { se obesidade })+(0,456 \text { se sobrepeso })+(0,582, \text { se } A F \text { de } D M)+}{(1,032, \text { se } A P \text { de } D M G)+(0,088 * \text { valor } G J)+(0,054 * \text { idade })]}
\end{gathered}
$$

AF: antecedente familiar, DM: diabetes mellitus, AP: antecedente pessoal, DMG: diabetes mellitus gestacional, GJ: glicemia de jejum

Com base na razão de chances, de acordo com o modelo, o histórico prévio de diabetes gestacional aumentou a chance de uso de insulina 2,8 vezes, a obesidade aumentou 2,3 vezes a chance de insulinoterapia, sobrepeso aumentou em 1,6 vezes e ter história familiar de DM aumentou essa chance 1,8 vezes. Cada ano na idade adicionou 1,05 a chance de uso de insulina durante a gestação, enquanto que cada unidade de valor de GJ aumentou a chance de terapia com insulina 1,09 vezes (Tabela 8).

Tabela 7 - Resultado do modelo de regressão logística multivariada para predição de insulinoterapia em gestantes com diabetes gestacional diagnosticada pela glicemia de jejum - HC-FMUSP - São Paulo, 2012-2015

\begin{tabular}{lcccc}
\hline \multicolumn{1}{c}{ Variável } & Coeficiente & Erro padrão & OR (IC 95\%) & Valor $\mathbf{p}$ \\
\hline Intercepto & $-12,23$ & 2,025 & & \\
Obesidade* $^{*}$ & 0,818 & 0,329 & $2,265(1,188-4,320)$ & 0,013 \\
Sobrepeso* $^{*}$ & 0,456 & 0,356 & $1,577(0,785-3,169)$ & 0,201 \\
História familiar de DM $^{\star \star}$ & 0,582 & 0,249 & $1,790(1,099-2,917)$ & 0,019 \\
DMG $^{1}$ prévio** & 1,032 & 0,349 & $2,807(1,418-5,559)$ & 0,003 \\
Idade (anos) $_{\text {Glicemia de jejum }(\mathbf{m g} / \mathbf{d L})^{2}}$ & 0,054 & 0,020 & $1,055(1,014-1,098)$ & 0,008 \\
\hline
\end{tabular}

1. DMG: diabetes mellitus gestacional, ${ }^{2}$. mg/dL: miligramas por decilitro, * Índice de massa corpórea normal e baixo peso como referência, ${ }^{* *}$ não apresentar tais históricos como referência 
Para facilitar a utilização na prática clínica do modelo preditivo de uso de insulina em gestantes diagnosticadas com DMG pela glicemia de jejum, construímos um nomograma com base no modelo descrito por Zee et al. ${ }^{(25)}$ (Figura 4).

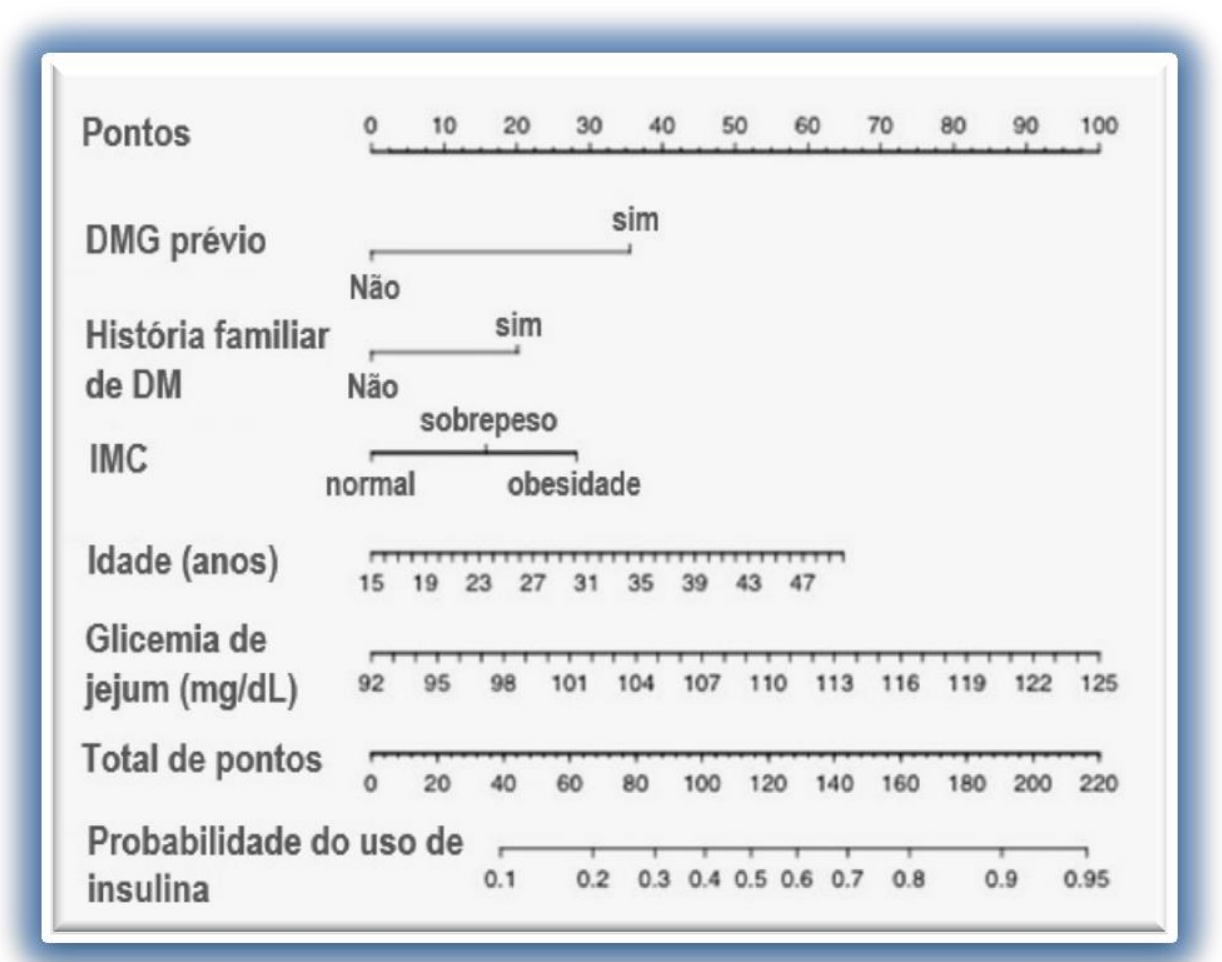

DMG: diabetes mellitus gestacional, DM: diabetes mellitus, IMC: índice de massa corpórea, $\mathrm{mg} / \mathrm{dL}$ : miligramas por decilitro

Figura 4 - Nomograma para predição do uso de insulina em gestantes com diabetes gestacional diagnosticadas pela glicemia de jejum antes de 24 semanas de gestação 


\subsubsection{Como usar o nomograma}

O modelo proposto no presente estudo identificou cinco variáveis para predição do uso de insulina. Cada uma dessas variáveis é representada em uma linha no nomograma, e uma pontuação respectiva é atribuída a cada uma delas.

Os pontos atribuídos para cada uma das variáveis são somados (TOTAL DE PONTOS), e é realizada a correlação desse total com uma estimativa final de probabilidade na linha abaixo no respectivo nomograma (PROBABILIDADE DO USO DE INSULINA).

Por exemplo, uma mulher com história de DMG prévio receberia 36 pontos, por ter antecedente familiar de DM2, pontuaria mais 20 pontos. Por ser obesa, ela receberia 28 pontos, se tivesse 35 anos, receberia mais 37 pontos e, com glicemia em jejum de $95 \mathrm{mg} / \mathrm{dL}$ no diagnóstico, receberia 9 pontos. Para esta mulher, a soma final seria de 130 pontos, que corresponde à probabilidade de $61 \%$ de necessidade de insulina durante o tratamento do DMG. Os pontos correspondentes a cada uma das variáveis estão expostos na Tabela 9. 
Tabela 8 - Pontuação correspondente a cada variável do nomograma

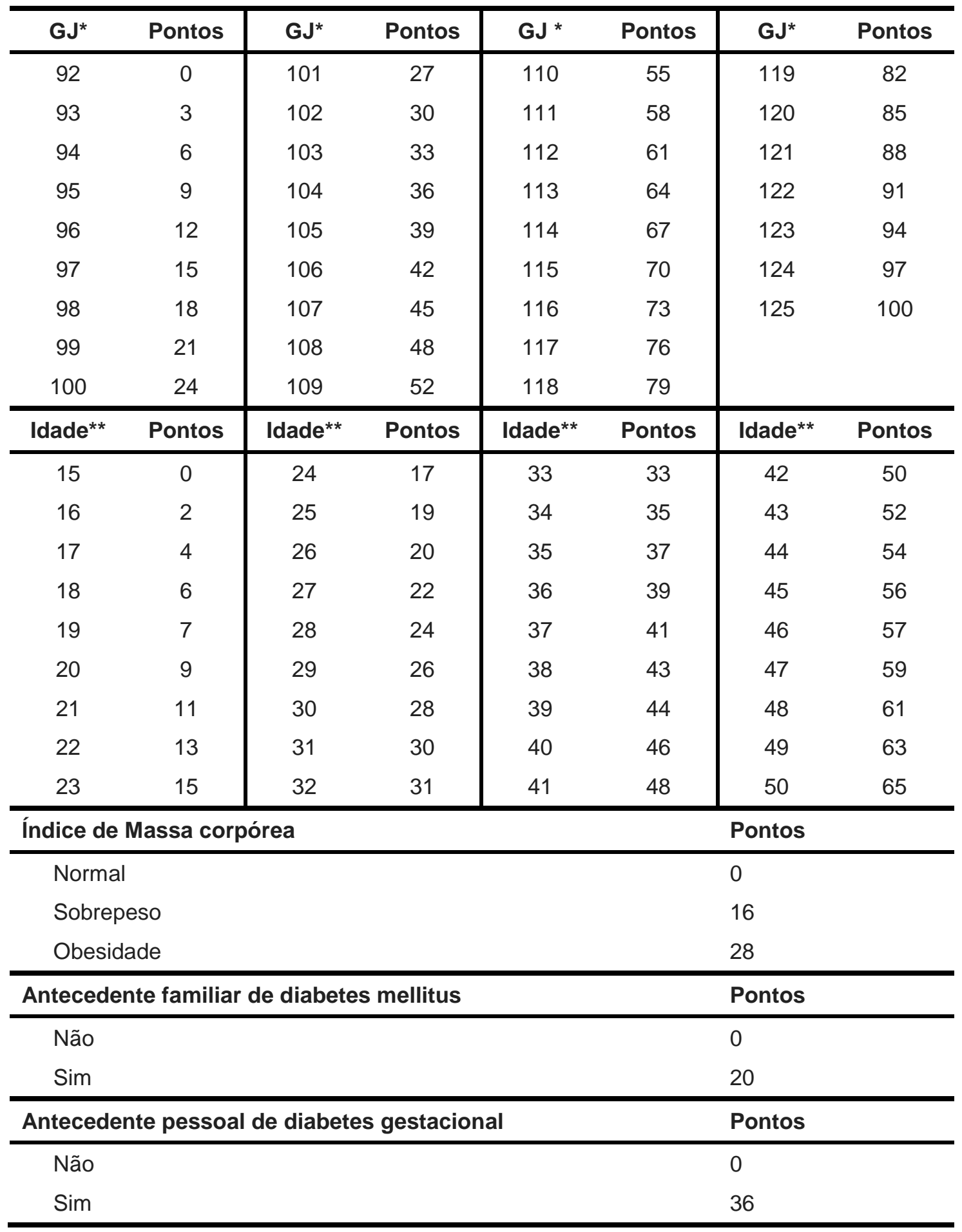

GJ: glicemia de jejum, * Glicemia de jejum em miligramas por decilitro, ** Idade em anos 
De modo a facilitar o uso clínico do nomograma, foi desenvolvida uma calculadora digital, que é uma alternativa ao uso do gráfico. Essa ferramenta está disponível no website: obstetricia.hc.fm.usp.br/CalcGDM. Na Figura 5, pode-se observar a aplicação da calculadora considerando-se o mesmo exemplo exposto para o uso do nomograma. 


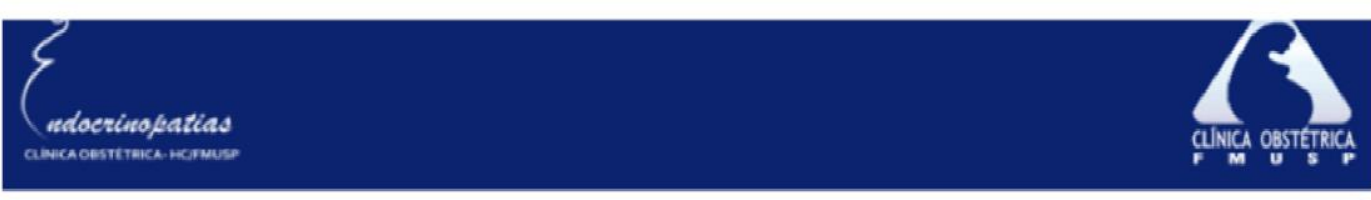

Calculadora da Probabilidade de Necessidade de Insulina em Pacientes com DMG Diagnosticado pela Glicemia de Jejum até 24 semanas

Dados obtidos de coorte retrospectiva de 408 mulheres com DMG diagnosticado pela glicemia de jejum maior que $92 \mathrm{mg} / \mathrm{dL}$ antes de 24 semanas de idade gestacional

(São Paulo, 2016)

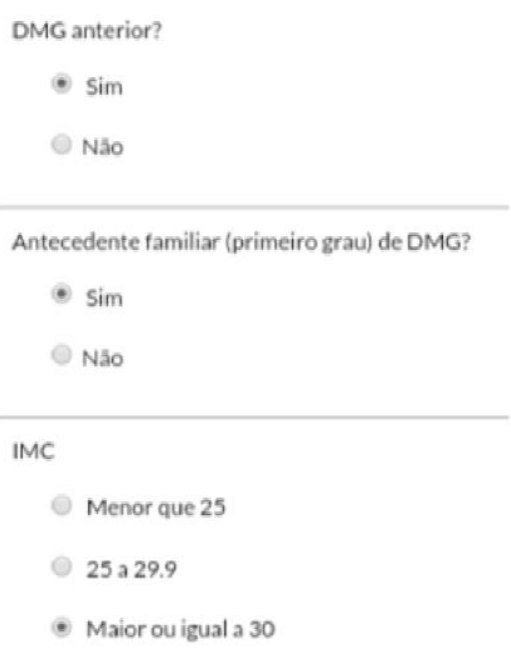

Idade (anos): $\quad 35$

Glicemia de jejum (mg/dL):

95

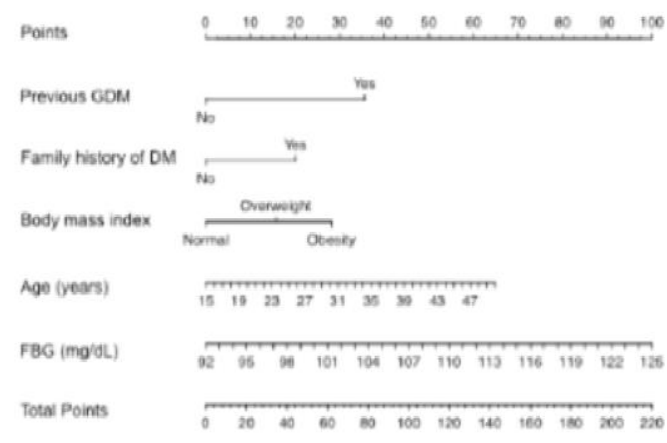

Probability of insulin need
A pontuação final é 130 pontos, o que representa uma probabilidade de $61 \%$ de necessidade do uso de insulina durante $o$ tratamento.

Figura 5 - Exemplo do uso da calculadora digital disponível no website: obstetricia.hc.fm.usp.br/CalcGDM para predição do uso de insulina durante a gestação em mulheres diagnosticadas com diabetes gestacional pela glicemia de jejum 


\subsubsection{Desempenho clínico do modelo preditivo do uso de insulina}

De acordo com a probabilidade de uso de insulina em gestantes com diagnóstico de DMG pela glicemia de jejum calculada pelo modelo preditivo proposto neste estudo, pretendeu-se classificar esse grupo de gestantes em dois grupos: "alto risco" para uso de insulina e "baixo risco" para necessidade de insulinoterapia.

A análise da curva ROC demonstrou área sob a curva de 0,74 IC 95\% 0,687-0,792 (Gráfico 3). Aplicando-se o método de validação cruzada leave one out, a acurácia de nosso modelo foi de $72,8 \%$.

Gráfico 1 - Curva ROC do modelo de predição do uso de insulinoterapia em gestantes com diabetes gestacional diagnosticada pela glicemia de jejum - HC-FMUSP - São Paulo, 2012-2015

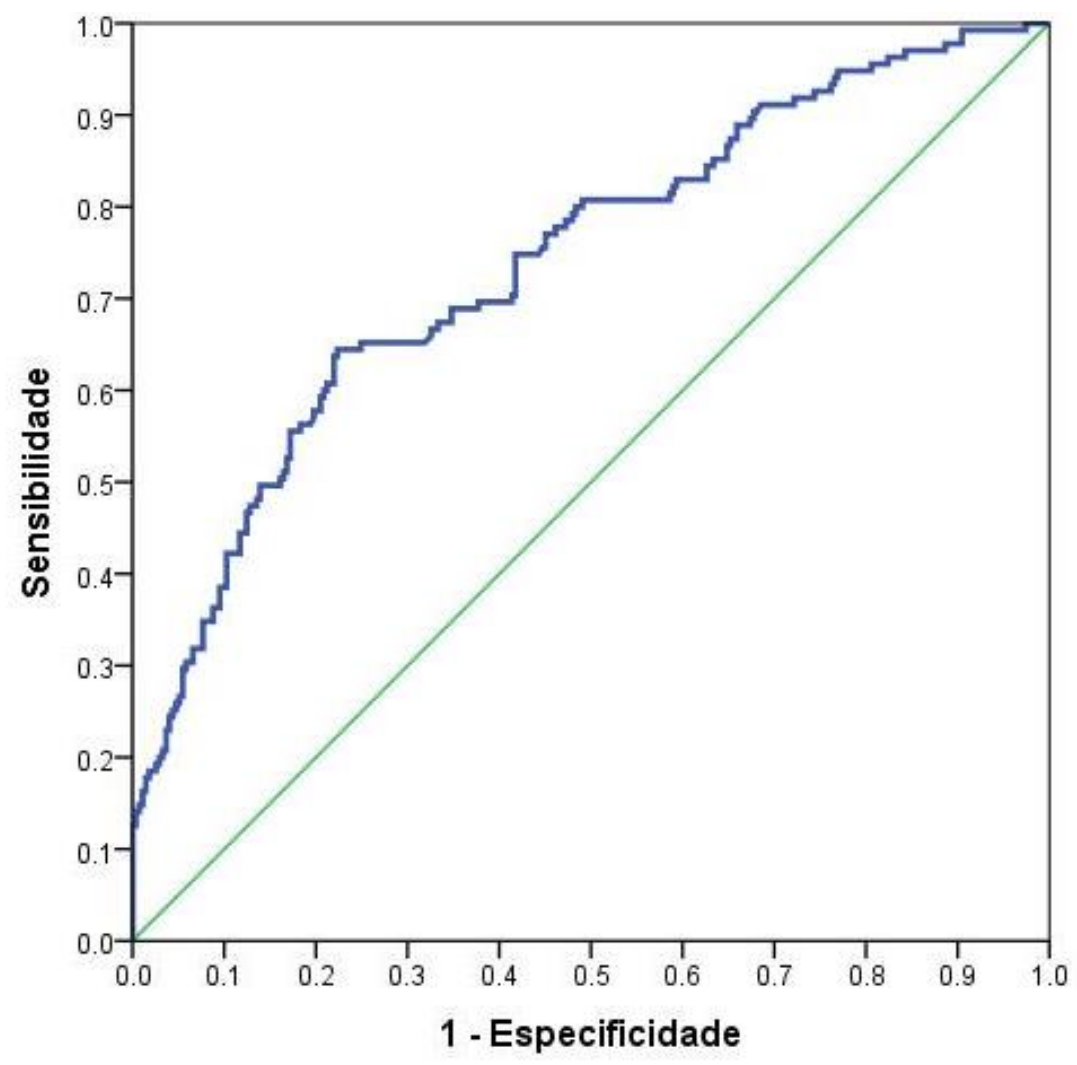


Utilizando-se o modelo proposto e assumindo-se probabilidades maiores ou iguais a 0,5 como "gestações de alto risco" e aquelas menores que 0,5 como "gestações de baixo risco", por meio da análise dessa curva, identifica-se sensibilidade de 34,8\% e especificidade de 91,6\% para predizer o uso de insulina em gestantes com DMG pela glicemia de jejum. Considerando-se essa classificação, o desempenho clínico do modelo foi estimado com VPP de 67,1\%, VPN de 74\% e acurácia de 73\% (Tabela 10).

Alternativamente, um corte de 0,3 para a probabilidade de uso de insulina corresponderia a uma sensibilidade de $67 \%$ e especificidade de $66 \%$ para essa classificação. Desta forma, utilizando-se o modelo proposto e assumindo probabilidades maiores ou iguais a 0,3 como "gestações de alto risco" e valores menores que 0,3 como "gestações de baixo risco", estimamos o desempenho clínico do modelo, com VPP de 49\%, VPN de $80 \%$ e acurácia de $66 \%$ (Tabela 10).

Tabela 9 - Desempenho do rastreamento para probabilidade do uso de insulina em gestantes com diabetes gestacional definido pelo modelo de regressão logística quando comparado com o uso real de insulina nas pacientes estudadas - HC-FMUSP - São Paulo, 2012-2015

\begin{tabular}{lcccccccc}
\hline $\begin{array}{l}\text { Probabilidade de } \\
\text { insulinoterapia }\end{array}$ & Dieta & Insulina & Total & Sen & Esp & VPP & VPN & Acu \\
\hline$<0,5$ (baixo risco) & 250 & 88 & 338 & & & & & \\
$\geq \mathbf{0 , 5}$ (alto risco) & 23 & 47 & 70 & $34,8 \%$ & $91,6 \%$ & $67,1 \%$ & $74,0 \%$ & $73,0 \%$ \\
TOTAL & 273 & 135 & 408 & & & & & \\
\hline$<\mathbf{0 , 3}$ (baixo risco) & 178 & 44 & 222 & & & & \\
$\geq \mathbf{0 , 3}$ (alto risco) & 95 & 91 & 186 & $67,0 \%$ & $66,0 \%$ & $49,0 \%$ & $80,0 \%$ & $66,0 \%$ \\
TOTAL & 273 & 135 & 408 & & & & & \\
\hline
\end{tabular}

Sen: sensibilidade, Esp: especificidade, VPP: valor preditivo positivo, VPN: valor preditivo negativo, Acu: acurácia. 


\section{DISCUSSÃO}

O aumento expressivo do número de casos de DMG na população mundial tem se imposto como um grande desafio obstétrico e de saúde pública. Por ser uma doença que se apresenta em largo espectro, das formas mais brandas às mais graves, modelos para identificação das pacientes que apresentarão maior dificuldade de controlar a hiperglicemia durante a gestação têm sido propostos na literatura. Tais estudos são importantes por permitirem pensarmos em adequação de condutas e recursos de acordo com a necessidade do caso. No entanto, até o momento, a literatura não apresentava nenhum modelo de estratificação de risco que avaliasse especificamente o grupo de gestantes que fazem diagnóstico de DMG no início da gestação pela glicemia de jejum, embora os modelos propostos já identificassem essa característica como fator de risco para maior gravidade do quadro.

Este estudo demonstrou que as gestantes diagnosticadas com DMG pela GJ no início da gestação e que necessitaram de insulinoterapia para o adequado controle glicêmico eram mais velhas, apresentavam maior IMC pré-gestacional e, mais comumente, hipertensão arterial, tinham gestações anteriores com maior frequência, assim como antecedente familiar de DM2, próprio de DMG e macrossomia anterior. A idade gestacional do diagnóstico não influenciou a necessidade de insulina, mas o valor da GJ no diagnóstico foi maior entre as gestantes que necessitaram de insulinoterapia.

Um modelo de predição de insulinoterapia nesse grupo de gestantes foi proposto considerando-se a idade materna, o IMC pré-gestacional, a história familiar de DM2 e a pessoal de DMG, assim como o valor da GJ no diagnóstico.

A seguir discutiremos nossos achados. 


\subsection{Características da população estudada}

Este estudo foi desenvolvido a partir de gestantes atendidas em um ambulatório de alto risco, em um serviço de atendimento terciário de saúde.

A indicação de corticoterapia para maturação pulmonar fetal em gestações de alto risco pode ser necessária e, de acordo com o protocolo assistencial do serviço, quando há essa necessidade, é indicada insulinoterapia concomitante, motivo pelo qual se optou por não incluir na análise aquelas que tiveram essa indicação.

Por serem oriundas de um ambulatório de alto risco, 32,4\% das pacientes elegíveis para o estudo apresentavam outra comorbidade. Essas gestantes foram encaminhadas para o nosso serviço para tratamento de outras doenças e também foram diagnosticadas com hiperglicemia na gestação. As doenças identificadas em nossa amostra foram hipertensão arterial, asma, lúpus eritematoso sistêmico e síndrome do ovário policístico. Duas dessas doenças apresentavam-se concomitantemente em $2 \%$ das pacientes. Chamou a atenção que $27,7 \%$ das gestantes fossem hipertensas, o que é considerado um fator de risco para o DMG ${ }^{(26)}$ e também um dos critérios propostos para o diagnóstico de síndrome metabólica ${ }^{(27)}$.

Outra característica que se destaca em nossa população é o fato de $77 \%$ das gestantes estarem acima do peso ideal no início da gestação, sendo que $48,3 \%$ eram obesas. A alta média de índice de massa corporal em nossas gestantes é fator de risco importante para o desenvolvimento de diabetes, assim como apontado por Shin et al. $(2015)^{(28)}$ e Mission et al. (2015) ${ }^{(29)}$.

A síndrome metabólica, uma epidemia mundial ${ }^{(27,30)}$, apresenta-se refletida também no perfil epidemiológico de nossas gestantes com altas frequências de hipertensão, obesidade e alteração no metabolismo da glicose. 


\subsection{Fatores de risco para uso de insulina em gestantes diagnosticadas com diabetes gestacional pela glicemia de jejum}

Avaliando-se os fatores de risco identificados na análise univariada para insulinoterapia em nossa população de gestantes, encontramos resultados semelhantes aos encontrados na literatura para gestantes com DMG, independentemente da forma de diagnóstico.

Ao se comparar o grupo de gestantes com controle adequado da hiperglicemia apenas com dieta com o grupo que fez uso de insulina, não nos surpreendeu identificarmos mulheres mais velhas, mais frequentemente obesas e hipertensas, no grupo com maior dificuldade de controle glicêmico. A resistência insulínica, que caracteriza a síndrome metabólica, manifesta-se em nossos achados.

O fato de o grupo que fez uso de insulinoterapia ter menos primigestas pode ter relação com a idade materna ser maior e, portanto, elas apresentarem mais frequentemente gestações anteriores.

Um dado que nos surpreendeu foi que, intuitivamente, esperava-se que o ganho de peso durante a gestação representasse fator de risco para formas mais graves de DMG, porém, isso não se confirmou ao compararmos o grupo Dieta e o grupo Insulina. Tal achado aponta que características associadas à síndrome metabólica antes da gestação são determinantes e sugere dois cuidados importantes: a orientação pré-concepcional, buscandose iniciar a gestação dentro do peso adequado, e que a identificação de fatores associados à síndrome metabólica pelo pré-natalista é um indicador de atenção à glicemia dessa gestante.

Observar ainda que, nos dois grupos avaliados, as gestantes ganharam peso de forma semelhante e adequada, cerca de $8 \mathrm{~kg}$ durante a gestação, sugere que em nosso pré-natal propomos um controle rigoroso da dieta e conseguimos uma boa adesão das gestantes. Mas que, a despeito do controle de ganho de peso e adoção de dieta adequada, uma porção dessas mulheres necessitou de insulina para o controle glicêmico, 
apontando para maior resistência insulínica nessas gestantes. Fatores relacionados à síndrome metabólica parecem colaborar nesse aspecto, de acordo com nossos achados.

O histórico de DMG ou de feto macrossômico na gestação anterior reforça a hipótese de que características pessoais de maior dificuldade do metabolismo da glicose relacionam-se à impossibilidade de controle glicêmico apenas com dieta na gestação. Se a idade é um fator que interfere na resistência à insulina, o DMG prévio demonstra sua presença em idade inferior, já que ocorreu em gestação anterior e, portanto, é esperado que em idade superior, na gestação atual, manifeste-se com maior gravidade. $O$ mesmo pensamento vale quanto à gestante que já apresentou repercussão fetal ao ter um filho com mais de $4.000 \mathrm{~g}$ ao nascimento, isto é, demonstra essa maior dificuldade de controlar a hiperglicemia durante a gestação.

A história familiar de diabetes mostrou-se como fator mais prevalente entre as gestantes que precisaram de insulinoterapia para adequado controle da hiperglicemia. Os fatores genéticos envolvidos na resistência insulínica e no diabetes reafirmam-se, destacando o valor da anamnese na primeira consulta de pré-natal e como fatores genéticos estão associados ao desenvolvimento do diabetes, seja na gestação como após o parto.

O valor da glicemia de jejum no diagnóstico também se mostrou como fator de risco para a necessidade de insulina na gestante com DMG diagnosticada pela GJ. Esse achado é concordante com os resultados apresentados na literatura ${ }^{(19,21)}$.

A idade gestacional no diagnóstico do DMG não foi uma variável significativa na análise univariada. Considerando-se que estamos em um serviço terciário e que, muitas vezes, a gestante demora a chegar até o primeiro atendimento, é relevante observar que a idade gestacional não interfere no prognóstico do tratamento quanto à necessidade de insulinoterapia. É importante verificar que a média do diagnóstico ocorreu ainda no primeiro trimestre, em média entre a $10^{\underline{a}}$ e a $11^{\text {a }}$ semana de gestação nos dois grupos avaliados e que, portanto, a diferença observada 
entre o valor da GJ entre os dois grupos e identificada como variável independente para o uso de insulina não pode ser relacionada às influências hormonais evolutivas da gestação.

\subsection{Modelos de predição para o uso de insulina na gestação em mulheres diagnosticadas com diabetes gestacional}

Após a análise univariada, as variáveis clínicas e laboratoriais com diferença significativa entre os grupos Dieta e Insulina foram ajustadas no modelo logístico. Aquelas que se mostraram preditoras independentes para insulinoterapia foram IMC pré-gravídico, história familiar de diabetes, história prévia de DMG, valor da GJ no diagnóstico e idade materna. Tais variáveis são semelhantes às de modelos logísticos já propostos pela literatura.

O estudo realizado por Sapienza et al. (2010) ${ }^{(17)}$ foi um estudo brasileiro, realizado em nosso serviço, e o primeiro modelo de predição de risco de insulinoterapia em gestantes com diabetes gestacional proposto na literatura.

Nesse estudo, obesidade e hipertensão foram frequentes na população estudada, à semelhança das características das gestantes incluídas em nosso estudo. A publicação de 2010 apresentava 67,2\% das gestantes com IMC pré-gestacional $\geq 30 \mathrm{~kg} / \mathrm{m}^{2}$ e $45,5 \%$ delas hipertensas e, neste estudo, realizado no mesmo serviço, observaram-se $48,3 \%$ de obesas e $27,7 \%$ de hipertensas.

Os autores desse estudo já apontavam em sua análise univariada os valores da GJ como preditores de insulinoterapia em mulheres com DMG, como também demonstrado em nosso estudo, porém, em seu modelo logístico de predição, essa variável não permaneceu. Obesidade prégestacional e história familiar de diabetes foram variáveis consideradas significativas e também encontradas no presente modelo. Variáveis preditoras encontradas no trabalho referido e não identificadas no nosso 
foram o número de valores alterados no TOTG e o valor da hemoglobina glicada. O TOTG não poderia ser avaliado no nosso trabalho, uma vez que as gestantes de nossa amostra foram diagnosticadas já pela GJ inicial, e por isso, dispensadas do teste. $\mathrm{O}$ valor da hemoglobina glicada pode variar de acordo com a idade gestacional devido a alterações gravídicas e, por isso, optamos por não avaliá-la em nosso estudo por falta de uniformidade entre as idades gestacionais de sua coleta, uma limitação de nosso estudo por sua característica retrospectiva.

Mendez-Figueroa et al. propuseram modelo de três variáveis em estudo americano de $2014^{(18)}$. Apesar de terem avaliado grupo de gestantes diferente da nossa amostra, ou seja, estudaram apenas gestantes com diagnóstico de DMG por valores alterados após sobrecarga de glicose e GJ normal pelo critério da ADA de $2004^{\left({ }^{(8)}\right.}$, o que chamaram de Mild DMG, o modelo proposto demonstrou aumento do uso de farmacoterapia para controle glicêmico. Quanto maior fosse o valor da glicemia de jejum no TOTG, menor a idade gestacional do diagnóstico e maior o IMC entre a 26 e a 30ạ semana de gestação. Ao discutir seus resultados, os autores declaram que acreditam que o diagnóstico antes de 20 semanas de gestação, provavelmente, representaria fator de risco para formas mais graves de DMG.

A característica ímpar de nosso estudo é justamente avaliar as gestantes diagnosticadas com DMG antes de 24 semanas de gestação pela GJ. Demonstramos que os valores da glicemia nesse exame no diagnóstico são, de fato, fatores preditores independentes para o uso de insulina para adequado controle da hiperglicemia, de acordo com os resultados de Mendez-Figueroa et al. ${ }^{(18)}$. O IMC maior como fator preditor para o uso de insulina está presente nos dois modelos, reforçando o envolvimento da síndrome metabólica na avaliação da gestante com DMG.

O terceiro modelo de predição de uso de insulina foi publicado na Austrália por Pertot et al. (2014) ${ }^{(19)}$. Nesse modelo de predição para uso de insulina em gestantes com DMG, mais uma vez a GJ foi apresentada como 
fator preditor independente da necessidade de insulina, reforçando a relevância do nosso estudo em se propor a estudar especificamente gestantes com alteração precoce desse exame. Assim como no modelo brasileiro de 2010 e no americano de 2014, o IMC também fez parte do modelo australiano, à semelhança do presente estudo. A história familiar de diabetes, assim como em nossos resultados, está presente no modelo proposto por Pertot et al. ${ }^{(19)}$. O valor da glicemia na primeira hora após sobrecarga e o valor da hemoglobina glicada são variáveis presentes nesse modelo, mas não avaliadas em nosso estudo para comparação. É interessante que os autores apontam em seus resultados que o diagnóstico de DMG após 25 semanas de gestação foi fator protetor para o uso de insulina $(R C$ 0,5). Dessa forma, reforçamos a importância do nosso estudo em se propor a estudar esse grupo de gestantes diagnosticadas com DMG antes de 24 semanas de gestação e com maior chance de apresentar formas graves da doença.

Entre os especialistas que assistem as gestantes com DMG, há um desejo de se identificar um valor da GJ capaz de estratificar o risco dessas mulheres e guiar o seguimento pré-natal. Todavia, nosso estudo demonstrou, à semelhança dos modelos já discutidos, o mesmo que Pertot et al. ${ }^{(19)}$ demonstram em seu estudo: que o valor isolado da glicemia de jejum não é suficiente para estratificar o risco do DMG e que variáveis clínicas têm importância significativa na equação.

O último modelo de predição de necessidade de insulinoterapia em gestantes com DMG descrito na literatura é o de Barnes et al. em 2016, também na Austrália. Em semelhança ao nosso estudo, o modelo proposto busca método de fácil aplicabilidade clínica.

As cinco variáveis identificadas em nosso modelo também foram usadas no modelo de sete variáveis do estudo australiano. Uma das variáveis não contemplada em nosso modelo foi o diagnóstico precoce de DMG antes de 24 semanas, o que se aplicaria a todas as gestantes avaliadas em nosso estudo. A outra variável é o valor da hemoglobina 
glicada, que optamos por não avaliar em nosso modelo, dada a disparidade de idade gestacional de coleta em nossa amostra, como já previamente sinalizado.

Um diferencial importante do estudo de Barnes et al. (21) em comparação aos modelos já apresentados é que foram os primeiros a validar o seu modelo em outra população, podendo demonstrar com clareza a aplicabilidade de seu modelo. Em nosso estudo, pela ausência de outra amostra para avaliação do modelo, optamos por utilizar a ferramenta estatística de validação leave one out ${ }^{(23)}$, que permite que o modelo seja testado na própria população que lhe originou, ao se propor a retirada de um indivíduo por vez da população que, então, torna-se uma nova população, e permite reaplicar o modelo. Isso é feito com todos os indivíduos, o que, em nosso caso, resultou em 408 aplicações diferentes do modelo, e os resultados foram comparados. Ao aplicarmos esse método de validação, o nosso modelo demonstrou acurácia de 72,8\%. Para evitar as limitações do estudo retrospectivo e validar nosso modelo em nova população, planejamos um estudo prospectivo para a aplicação do modelo.

Alguns pontos da metodologia do estudo australiano podem ser discutidos. Ao avaliar uma população entre 1999 e 2015, foram comparadas gestantes diagnosticadas por critérios diferentes, inicialmente propostos em 1991 e reavaliados em 1998. Para tornar o modelo de fácil aplicabilidade clínica, Barnes et al. ${ }^{(21)}$ dicotomizaram suas sete variáveis e as compararam como iguais em relevância para o resultado final do modelo. Estatisticamente, isso é bastante questionável, uma vez que cada uma das variáveis interfere no risco de insulinoterapia de maneira particular, como pudemos observar pelos coeficientes de cada variável encontrados em nosso modelo logístico. Por exemplo, enquanto em nosso modelo o coeficiente correspondente a ter história de DMG prévio é de 1,032 o de ter história familiar de DM2 é de quase sua metade, de 0,582. O coeficiente de cada ano na idade materna é de 0,054 , o que representa diferença importante ao se comparar uma gestante de 20 anos e outra de 38 anos. Colocá-los simplesmente como iguais no modelo como propõe Barnes et al. 
(21) é desconsiderar a influência característica de cada variável em predizer o risco de insulinoterapia e questionar a validade estatística.

A alternativa que utilizamos em nosso estudo para facilitar a aplicação clínica e evitar erros estatísticos foi desenvolver o nomograma e a calculadora digital. Ambos utilizam o peso de cada uma das variáveis do modelo sem aumentar a dificuldade do uso do método. O nomograma pode facilmente ser impresso e deixado nos consultórios em que ocorrem atendimentos de pré-natal ou acessado pelos smartphones ou internet tão difundidos em nosso meio. Acreditamos que a calculadora digital seja o método preferido do usuário ao utilizar os meios digitais e uma ferramenta atraente para o médico que assistir essas gestantes.

Dessa forma, o modelo de predição para uso de insulina proposto neste estudo tem potencial de grande importância clínica, ao representar uma ferramenta para reconhecer mulheres com formas de hiperglicemia mais graves entre as gestantes precocemente diagnosticadas com DMG, orientar encaminhamentos para serviços de referência especializados ou redirecionar às unidades básicas aquelas gestantes identificadas como baixo risco para formas graves da doença e, dessa forma, permitir o uso racional dos recursos ao alocar mais adequadamente as gestantes com DMG de acordo com suas necessidades.

Outra crítica ao estudo australiano de 2016 cabe à proposta de estratificação de risco a partir do modelo encontrado. Barnes et al. consideraram de alto risco para insulinoterapia as gestantes que apresentarem seis ou todas as sete variáveis de risco apontadas pelo modelo e de baixo risco aquelas com nenhum ou apenas um dos fatores de risco. Aquelas com dois a cinco fatores de risco, que representaram $72 \%$ de sua amostra, foram consideradas de médio risco, para quem propuseram monitoramento mais próximo pelo maior risco de eventos adversos na gestação. Portanto, seu modelo não estabelece se mais da metade da população estudada é de alto ou baixo risco. Pensando-se na aplicabilidade 
clínica, fica indefinido, na maioria das vezes, para quem vai assistir a paciente como fazer o seguimento clínico.

Acreditamos que decidir qual seria o ponto de corte do modelo para determinar quais são as gestantes consideradas de baixo ou alto risco depende do objetivo da utilização do modelo. Ao se optar por sensibilidades maiores, deixaremos de tratar formas graves de DMG com menos frequência, mas manteremos mais gestantes em serviço especializado e utilizando mais recursos. Ao se optar por especificidades maiores, mais gestantes seriam contrarreferenciadas aos serviços de atenção primária, priorizando atendimento especializado apenas para casos de maior risco, mas potencialmente deixando de identificar gestantes que vão demandar insulinoterapia para controle glicêmico. Enquanto 0 modelo ideal apresentaria tanto especificidade e sensibilidade altas, observamos que nosso modelo teve desempenho semelhante aos descritos na literatura, cabendo a nós a reflexão de como utilizá-lo de acordo com a realidade de nossos serviços, se optaremos pelo ponto de corte de 0,3 ou 0,5 para definir as gestantes que serão de baixo ou alto risco e racionalizarmos a utilização de nossos recursos da melhor maneira.

Sapienza et al. (17) propuseram ponto de corte para risco de insulinoterapia de 0,3 , e as gestantes com risco acima desse ponto foram consideradas de alto risco. No modelo dos autores, considerando uma primeira consulta de pré-natal em que seria dado o diagnóstico de DMG e, portanto, a hemoglobina glicada ainda não estaria disponível, a acurácia encontrada foi de 66,4\%, enquanto ao se acrescentar o valor da hemoglobina glicada à equação, simulando a segunda consulta após o diagnóstico, de retorno com exames, a acurácia foi de 65,6\%. Em nosso modelo, assumindo o mesmo ponto de corte de 0,3, a acurácia calculada foi de $66 \%$, em consonância com os resultados encontrados no estudo brasileiro.

A avaliação do desempenho do modelo de Mendez-Figueroa et al. ${ }^{(18)}$ apresentou área sob a curva $\mathrm{ROC}$ de 0,70 e, optando-se por um ponto de 
corte de 4, VPP de $48 \%$ e VPN de 78\%. Nosso estudo apresentou desempenho semelhante, com área sob a curva de 0,74 e, para o corte de 0,3, VPP de $49 \%$ e VPN de $80 \%$

Pertot et al. ${ }^{(19)}$ optaram por apresentar um ponto de corte de 0,5, abaixo do qual as gestantes do estudo foram chamadas de baixo risco. Nesse caso, o modelo apresentava desempenho de $66 \%$ de sensibilidade e $37 \%$ de especificidade. Para o mesmo ponto de corte de 0,5 , nosso modelo apresenta sensibilidade de $35 \%$ e especificidade de $92 \%$, ou seja, o modelo australiano está mais propenso a falsos positivos e o nosso, a falsos negativos para o ponto de corte escolhido.

\subsection{Considerações finais}

O presente estudo avaliou de forma inédita um grupo específico de gestantes diagnosticadas com DMG, aquelas com GJ alterada pelos critérios da IADPSG antes de 24 semanas de gestação.

Uma limitação deste trabalho é o fato de nossa amostra ser oriunda de um serviço terciário de atendimento pré-natal, o que faz com que muitas de nossas gestantes não tenham apenas o diagnóstico de DMG, mas também outros que motivaram seu encaminhamento ao serviço, além de ser um estudo retrospectivo em que encontramos limitações, como a dificuldade de avaliar o desempenho da hemoglobina glicada no modelo pela disparidade de idades gestacionais da sua coleta.

Os resultados encontrados estão em concordância com os já apresentados previamente na literatura e demonstram de maneira mais consistente a glicemia de jejum antes de 24 semanas de gestação como preditor independente para a necessidade de insulinoterapia e que caracteriza um grupo de gestantes com DMG diferente do grupo das diagnosticadas mais tardiamente pelo TOTG. 
Tendo em vista que, até o momento, poucos estudos foram conduzidos com a proposta de sugerir modelos de predição de risco em gestantes diagnosticadas com DMG e que o nosso modelo propôs-se a avaliar um grupo que impacta significativamente nas medidas de saúde pública para diagnóstico e manejo da doença, estudos para validar a aplicabilidade desses modelos são fundamentais para guiar protocolos terapêuticos de acordo com as diferentes realidades econômicas e sociais, dando perspectivas de aprimoramento do cuidado a essas gestantes, mesmo com recursos escassos, e alento a tantos profissionais que se propõe a cuidar delas diante de tantos obstáculos encontrados na escassez de recursos. 
6 CONCLUSÃO 


\section{CONCLUSÃO}

Idade materna, IMC pré-gestacional, história familiar de diabetes, história pessoal de DMG e valor da glicemia de jejum no diagnóstico são fatores preditores independentes para a necessidade de insulinoterapia em mulheres diagnosticadas com DMG pela glicemia de jejum antes de 24 semanas de gestação. Este estudo possibilitou o desenvolvimento de um modelo de predição de risco para insulinoterapia nesse grupo de mulheres e a elaboração de um nomograma e uma calculadora digital de modo a facilitar o seu uso clínico. 


\section{REFERÊNCIAS}




\section{REFERÊNCIAS}

1. World Health Organization. [Internet] Diagnostic criteria and classification of hyperglycaemia first detected in pregnancy: a World Health Organization guidelin. 2013. [acessado 7 de setembro de 2017]. Disponível em:http://apps.who.int/iris/bitstream/10665/85975/1/WHO_NMH_MND_13.2_ eng

.2. Metzger BE, Lowe LP, Dyer AR, Trimble ER, Chaovarindr U, Coustan DR, et al. Hyperglycemia and adverse pregnancy outcomes. $N$ Engl J Med. 2008;358(19):1991-2002.

3. Organização Pan-Americana da Saúde, Ministério da Saúde, Federação Brasileira das Associações de Ginecologia e Obstetrícia, Sociedade Brasileira de Diabetes. [Internet] Rastreamento e diagnóstico de diabetes mellitus gestacional no Brasil. 2017. [acessado 10 de março de 2018]. Disponível em: http://iris.paho.org/xmlui/bitstream/handle/123456789/34278/9788579671180 -por.pdf?sequence $=1$ \&isAllowed=y

4. Hod M, Kapur A, Sacks DA, Hadar E, Agarwal M, Di Renzo GC, et al. The International Federation of Gynecology and Obstetrics (FIGO) Initiative on gestational diabetes mellitus: A pragmatic guide for diagnosis, management, and care. Int J Gynaecol Obstet. 2015;131 Suppl 3:S173-211.

5. O'Sullivan JB, Mahan CM. Criteria for the oral glucose tolerance test in pregnancy. Diabetes. 1964;13:278-85.

6. Bhavadharini B, Uma R, Saravanan P, Mohan V. Screening and diagnosis of gestational diabetes mellitus - relevance to low and middle income countries. Clin Diabetes Endocrinol. 2016;2:13.

7. Carpenter MW, Coustan DR. Criteria for screening tests for gestational diabetes. Am J Obstet Gynecol. 1982;144(7):768-73. 
8. Diagnosis and classification of diabetes mellitus. Diabetes Care. 2004;27 Suppl 1:S5-s10.

9. Metzger BE, Gabbe SG, Persson B, Buchanan TA, Catalano PA, Damm $P$, et al. International association of diabetes and pregnancy study groups recommendations on the diagnosis and classification of hyperglycemia in pregnancy. Diabetes Care. 2010;33(3):676-82.

10. ACOG Committee Opinion No. 435: postpartum screening for abnormal glucose tolerance in women who had gestational diabetes mellitus. Obstet Gynecol. 2009;113(6):1419-21.

11. Schmidt MI, Duncan BB, Reichelt AJ, Branchtein L, Matos MC, Costa e Forti A, et al. Gestational diabetes mellitus diagnosed with a 2-h 75-g oral glucose tolerance test and adverse pregnancy outcomes. Diabetes Care. $2001 ; 24(7): 1151-5$.

12. Trujillo J, Vigo A, Duncan BB, Falavigna M, Wendland EM, Campos MA, et al. Impact of the International Association of Diabetes and Pregnancy Study Groups criteria for gestational diabetes. Diabetes Res Clin Pract. 2015;108(2):288-95.

13. Liao S, Mei J, Song W, Liu Y, Tan YD, Chi S, et al. The impact of the International Association of Diabetes and Pregnancy Study Groups (IADPSG) fasting glucose diagnostic criterion on the prevalence and outcomes of gestational diabetes mellitus in Han Chinese women. Diabet Med. 2014;31(3):341-51.

14. Alunni ML, Roeder HA, Moore TR, Ramos GA. First trimester gestational diabetes screening - Change in incidence and pharmacotherapy need. Diabetes Res Clin Pract. 2015;109(1):135-40. 
15. Meshel S, Schejter E, Harel T, Maslovitz S, Germez N, Elimelech B, et al. Can we predict the need for pharmacological treatment according to demographic and clinical characteristics in gestational diabetes? J Matern Fetal Neonatal Med. 2016;29(13):2062-6.

16. Bakiner O, Bozkirli E, Ozsahin K, Sariturk C, Ertorer E. Risk factors that can predict antenatal insulin need in gestational diabetes. J Clin Med Res. 2013;5(5):381-8.

17. Sapienza AD, Francisco RP, Trindade TC, Zugaib M. Factors predicting the need for insulin therapy in patients with gestational diabetes mellitus. Diabetes Res Clin Pract. 2010;88(1):81-6.

18. Mendez-Figueroa H, Daley J, Lopes VV, Coustan DR. Predicting the need for medical therapy in patients with mild gestational diabetes. Am $J$ Perinatol. 2014;31(2):105-12.

19. Pertot T, Molyneaux L, Tan K, Ross GP, Yue DK, Wong J. Can common clinical parameters be used to identify patients who will need insulin treatment in gestational diabetes mellitus? Diabetes Care. 2011;34(10):22146.

20. Hoffman L, Nolan C, Wilson JD, Oats JJ, Simmons D. Gestational diabetes mellitus - management guidelines. The Australasian Diabetes in Pregnancy Society. Med J Aust. 1998;169(2):93-7.

21. Barnes RA, Wong T, Ross GP, Jalaludin BB, Wong VW, Smart CE, et al. A novel validated model for the prediction of insulin therapy initiation and adverse perinatal outcomes in women with gestational diabetes mellitus. Diabetologia. 2016;59(11):2331-8.

22. Zugaib M, Bittar R, Francisco R. Protocolos Assistenciais. $5^{\mathrm{a}}$ edição ed. Atheneu 2015. 
23. Kuhn M. [Internet]. Futility Analysis in the cross-validation of machine learning models 2014 [acessado 11 de agosto de 2016]. Disponível em: https://arxiv.org/pdf/1405.6974v1.pdf.

24. Kuhn M. [Internet]. Caret: Classification and Regression Training 2016 [acessado 11 de agosto de 2016]. Disponível em: https://CRAN.Rproject.org/package=caret.

25. Van Zee KJ, Manasseh DM, Bevilacqua JL, Boolbol SK, Fey JV, Tan LK, et al. A nomogram for predicting the likelihood of additional nodal metastases in breast cancer patients with a positive sentinel node biopsy. Ann Surg Oncol. 2003;10(10):1140-51.

26. Leon MG, Moussa HN, Longo M, Pedroza C, Haidar ZA, MendezFigueroa $\mathrm{H}$, et al. Rate of gestational diabetes mellitus and pregnancy outcomes in patients with chronic hypertension. Am $J$ Perinatol. 2016;33(8):745-50.

27. Samson SL, Garber AJ. Metabolic syndrome. Endocrinol Metab Clin North Am. 2014;43(1):1-23.

28. Shin D, Song WO. Prepregnancy body mass index is an independent risk factor for gestational hypertension, gestational diabetes, preterm labor, and small- and large-for-gestational-age infants. J Matern Fetal Neonatal Med. 2015;28(14):1679-86.

29. Mission JF, Marshall NE, Caughey AB. Pregnancy risks associated with obesity. Obstet Gynecol Clin North Am. 2015;42(2):335-53.

30. O'Neill S, O'Driscoll L. Metabolic syndrome: a closer look at the growing epidemic and its associated pathologies. Obes Rev. 2015;16(1):112. 